\title{
$1 \quad$ Neural Correlates of Optimal Multisensory Decision Making
}

$5{ }^{1}$ Institute of Neuroscience, Key Laboratory of Primate Neurobiology, CAS Center for Excellence

6 in Brain Science and Intelligence Technology, Chinese Academy of Sciences, Shanghai, China

$7 \quad{ }^{2}$ University of Chinese Academy of Sciences, Beijing, China

$8 \quad{ }^{3}$ University of Geneva, Geneva, Switzerland

9

$10 *$ These authors contributed equally to this work.

11 \# These senior authors contributed equally to this work.

$12 \S$ Correspondence should be addressed to: Yong Gu (guyong@ion.ac.cn) 


\section{Abstract}

16 Perceptual decisions are often based on multiple sensory inputs whose reliabilities rapidly vary

17 over time, yet little is known about how our brain integrates these inputs to optimize behavior. Here

18 we show multisensory evidence with time-varying reliability can be accumulated near optimally,

19 in a Bayesian sense, by simply taking time-invariant linear combinations of neural activity across

20 time and modalities, as long as the neural code for the sensory inputs is close to an invariant linear

21 probabilistic population code (ilPPC). Recordings in the lateral intraparietal area (LIP) while

22 macaques optimally performed a vestibular-visual multisensory decision-making task revealed that

23 LIP population activity reflects an integration process consistent with the ilPPC theory. Moreover,

24 LIP accumulates momentary evidence proportional to vestibular acceleration and visual velocity

25 which are encoded in sensory areas with a close approximation to ilPPCs. Together, these results

26 provide a remarkably simple and biologically plausible solution to optimal multisensory decision

27 making.

28

29 Keywords: Perceptual decision making, multisensory integration, LIP, probabilistic population

30 code, vestibular, optic flow, self-motion perception 


\section{$31 \quad$ Introduction}

32 Most perceptual decisions are based on multiple sensory inputs whose reliabilities vary over time.

33 For instance, a predator can rely on both auditory and visual information to determine when and

34 where to strike a prey, but these two sources of information are not generally equally reliable, nor

35 are their reliabilities constant over time: as the prey gets closer, the quality of the image and sound

36 typically improves, thus increasing their reliabilities. Although such multisensory decision making

37 happens frequently in the real world, the underlying neural mechanisms remain largely unclear.

39 The so-called drift-diffusion model (DDM) ( ${ }^{1}$ Ratcliff, 1978; ${ }^{2}$ Ratcliff and McKoon, 2008;

$40 \quad{ }^{3}$ Ratcliff and Rouder, 1998; ${ }^{4}$ Ratcliff and Smith, 2004), a widely used model of perceptual

41 decision making, cannot deal with such decisions optimally in its most standard form. DDMs have

42 been shown to implement the optimal policy for decisions involving just one source of sensory

43 evidence whose reliability is constant over time ( ${ }^{5}$ Laming, 1968; ${ }^{6}$ Bogacz, et al., 2006). Under

44 such conditions, DDMs can implement the optimal strategy by simply summing evidence over time

45 until an upper or lower bound, corresponding to the two possible choices, is hit ( ${ }^{6}$ Bogacz, et al.,

46 2006). This type of models lends itself to a straightforward neural implementation in which neurons

47 simply add their sensory inputs until they reach a preset threshold ( ${ }^{2}$ Ratcliff and McKoon, 2008;

$48 \quad{ }^{7}$ Gold and Shadlen, 2007).

50 When multiple sensory inputs are involved, the standard DDMs can accumulate sensory evidence

51 optimally as long as the reliabilities of the evidence stay constant during a single trial and across

52 trials. Under this scenario, optimal integration of evidence over time can be achieved by first taking

53 a weighted sum of the momentary evidence at each time step, with weights proportional to the

54 reliability of each sensory stream, followed by temporal integration ( ${ }^{8}$ Drugowitsch, et al., 2014).

55 However, this strategy no longer works when the reliabilities change over time within a single trial. 
56 In this case, the momentary evidence must be linearly combined with weights proportional to the

57 time-varying reliabilities, which requires that the synaptic weights change on a very fast time scale

58 since, in the real life, reliability can change significantly over tens of milliseconds. Moreover, when

59 the reliabilities of the sensory inputs are not known in advance, which is typically the case in real-

60 world situations, neurons cannot determine how to appropriately modulate their synaptic weights

61 until after the sensory inputs have been observed. Therefore, even if it is possible to extend standard

62 DDMs to time-varying reliability ( ${ }^{8}$ Drugowitsch, et al., 2014), it is unclear how such a solution

63 could be implemented biologically.

65 In contrast, there exists another class of models which does not necessarily involve changes in 66 synaptic strength. As long as the sensory inputs are encoded with what is known as "invariant linear

67 probabilistic population codes" (ilPPC), the neural solution for optimal multisensory integration is

68 remarkably simple: it only requires that neurons compute linear combinations of their inputs across

69 time or modalities using fixed-reliability-independent—synaptic weights ( ${ }^{9}$ Beck, et al., 2008;

$70{ }^{10} \mathrm{Ma}$, et al., 2006). This solution relies on one specific property of ilPPC: the reliability of the

71 neural code is proportional to the amplitude of the neural responses. As a result, when summing

72 two sensory inputs with unequal reliability, the sensory input with the lowest reliability contribute

73 less to the sum because of its lower firing rate. This is formally equivalent to weighting Gaussian

74 samples with their reliability in an extended DDM, except that there is no need for actual weight

75 changes with ilPPC $\left({ }^{10} \mathbf{M a}\right.$, et al., 2006). Hence, the ilPPC framework is a promising solution to

76 multisensory decision-making tasks, but it lacks physiological supports.

78 To investigate whether the brain may implement this solution, we recorded the activity of single

79 neurons in the lateral intraparietal area (LIP) in macaques trained to discriminate their heading

80 direction of self-motion based on multiple sensory inputs: vestibular signals, visual optic flow, or

81 both. Importantly, the vestibular and visual stimuli followed a Gaussian-shape velocity temporal 
82 profile, producing naturally varied cue reliability over time within each trial. This behavioral

83 paradigm has been well-established for studying multisensory heading discrimination in the past

84 decade $\left({ }^{11}\right.$ Fetsch, et al., 2012; ${ }^{12} \mathrm{Gu}$, et al., 2008; ${ }^{13}$ Fetsch, et al., 2013). Nevertheless, these

85 previous studies focused on areas that encode momentary heading inputs, leaving it unknown how

86 these sensory inputs are further accumulated by downstream neurons (e.g. LIP) during perceptual

87 decision making.

89 We focus first in LIP because it is the most extensively studied brain region where buildup choice-

90 related activity has been found during visuomotor decisions in macaques $\left(^{7}\right.$ Gold and Shadlen,

$912007 ;{ }^{14}$ Shadlen and Newsome, 2001; ${ }^{15}$ Shadlen and Newsome, 1996; ${ }^{16} \mathrm{Huk}$, et al., 2017;

$92{ }^{17}$ Roitman and Shadlen, 2002). In addition, LIP receives abundant anatomical inputs

93 ( ${ }^{18}$ Boussaoud, et al., 1990) from areas encoding momentary vestibular and visual self-motion

94 information for heading discrimination, such as the dorsal medial superior temporal (MSTd) area

$95\left({ }^{12} \mathrm{Gu}\right.$, et al., 2008; ${ }^{19} \mathrm{Gu}$, et al., 2006) and the ventral intraparietal area (VIP) $\left({ }^{20} \mathrm{Chen}\right.$, et al., 2011c;

$96{ }^{21}$ Chen, et al., 2013). It is therefore expected that the activity of LIP neurons should carry buildup

97 choice signals germane to the formation of multisensory decisions. Note that two recent rodent

98 studies ( $\left({ }^{22}\right.$ Nikbakht, et al., 2018; ${ }^{23}$ Raposo, et al., 2014) also have described multisensory decision

99 signals in rat posterior parietal cortex, a region analogous to its primate counterpart. However, these

100 studies did not characterize the computational solution implemented by these neural circuits, which

101 is precisely the question we investigate here. Specifically, we explored whether the response of LIP

102 neurons is consistent with the ilPPC theory in which neurons take fixed linear combinations of their

103 sensory inputs without any need for complex, time-dependent, modality-specific, reweighting of

104 the sensory inputs during multisensory decision making. 


\section{Results}

\section{Optimal multisensory decision-making behavior on macaques}

107 We trained two macaque monkeys to perform a vestibular-visual multisensory decision-making 108 task $\left({ }^{12} \mathbf{G u}\right.$, et al., 2008) (Figure 1a). On each trial, the monkeys experienced a 1.5 s-fixed-duration

109 forward motion with a small deviation either to the left or to the right of the dead ahead. At the end

110 of the trial, the animals were required to report the perceived heading direction by making a saccade

111 decision to one of the two choice targets (Figure 1b). We randomly interleaved three cue conditions

112 over trials: a vestibular condition and a visual condition in which heading information was solely

113 provided by inertial cues and optic flow, respectively, and a combined condition consisting of

114 congruent vestibular and visual cues. Importantly, both the vestibular and visual stimuli followed a

115 Gaussian-shape velocity temporal profile, peaking at the middle of the 1.5 -s stimulus duration. This

116 modulation of velocity over time has an important implication for the reliability of the sensory

117 inputs provided to the animals. Indeed, previous psychophysical studies have established that a

118 model in which the reliability of the visual flow field is proportional to velocity and the reliability

119 of the vestibular signal is proportional to the acceleration, provides the best fits to the behavioral

120 data $\left({ }^{8}\right.$ Drugowitsch, et al., 2014). Therefore, this stimulus allows us to test how neural circuits

121 accumulate multisensory evidence whose reliability varies over time with distinct temporal profiles

122 (see below).

124 To quantify the monkeys' behavioral performance, we plotted psychometric curves for each cue 125 condition (Figure 1c). Consistent with the previous results $\left({ }^{12} \mathbf{G u}\right.$, et al., 2008), the monkeys made 126 more accurate decisions in the combined condition, as evidenced by a steeper psychometric 127 function and a smaller psychophysical threshold (Figure 1c). Across all recording sessions and for 128 both monkeys, the psychophysical threshold of the combined condition was significantly smaller 129 than those of single cue conditions and close to the threshold predicted by optimal Bayesian 
a
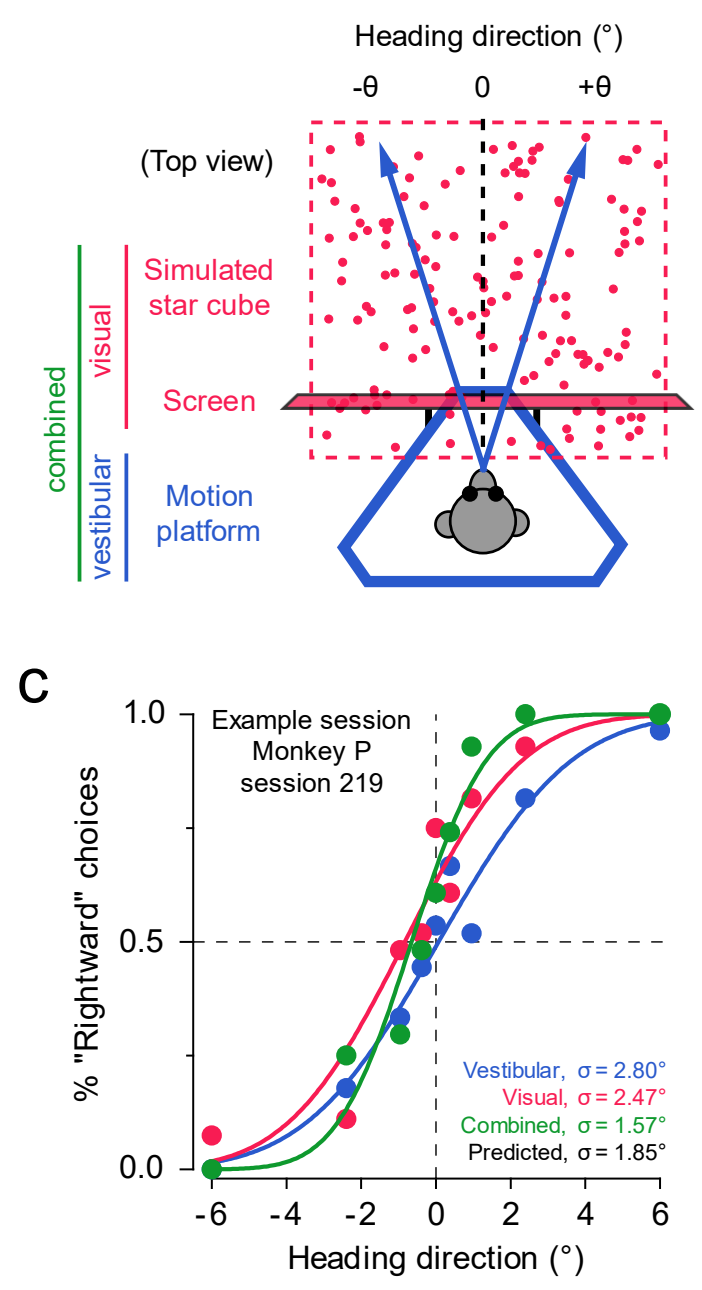

b

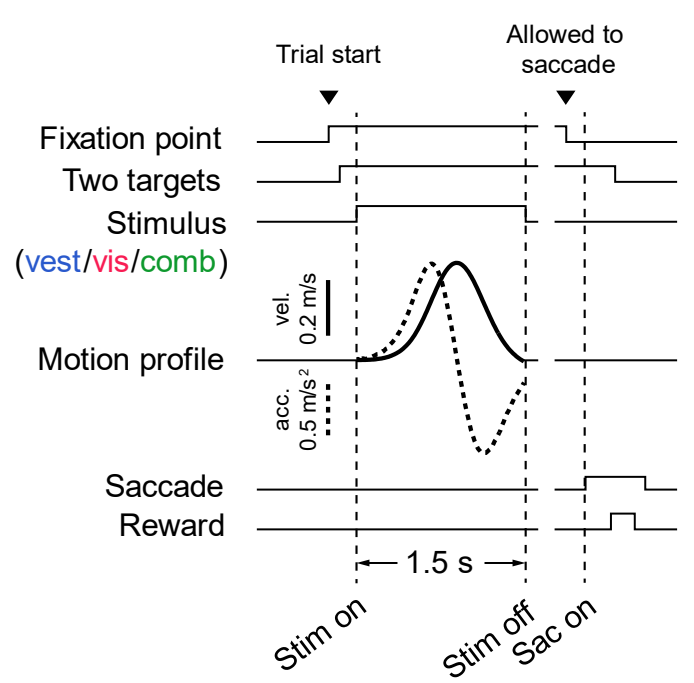

d

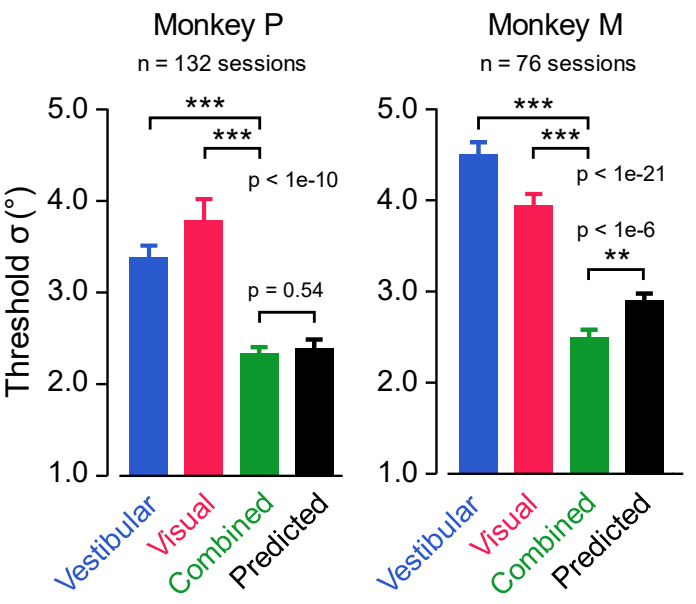

Figure 1 Optimal cue integration in vestibular-visual multisensory decision-making task.

(a) Schematic drawing of the experimental setup (top view). The vestibular (blue) and visual (red) stimuli of self-motion were provided by a motion platform and an LCD screen mounted on it, respectively. The monkey was seated on the platform and physically translated within the horizontal plane (blue arrows), whereas the screen rendered optic flow simulating what the monkey would see when moving through a three-dimensional star field (red dots). In a combined condition (green), both vestibular and visual stimuli were presented synchronously. The monkey's task was to discriminate whether the heading direction was to the left or the right of the straight ahead (black dashed line). (b) Task timeline. The monkey initiated a trial by fixating at a fixation point, and two choice targets appeared. The monkey then experienced a 1.5-s forward self-motion stimulus with a small leftward or rightward component, after which the monkey reported his perceived heading by making a saccadic eye movement to one of the two targets. The self-motion speed followed a Gaussian-shape profile. (c) Example psychometric functions from one session. The proportion of "rightward" choices is plotted against the headings for three cue conditions respectively. Smooth curves represent best-fitting cumulative Gaussian functions. (d) Average psychophysical thresholds from two monkeys for three conditions and predicted thresholds calculated from optimal cue integration theory (black bars). Error bars indicate s.e.m.; $p$ values were from paired t-test. 
131 multisensory integration $\left({ }^{24}\right.$ Knill and Richards, 1996) (Figure 1d). Therefore, the monkeys can

132 integrate vestibular and visual cues near-optimally during our multisensory decision-making task.

134 Heterogeneous multisensory choice signals in LIP

135 Next, we set out to explore how these optimal decisions were formed in the brain. We recorded 136 from 164 single, well-isolated neurons in LIP of two monkeys while they were performing the task

137 (Supplementary Figure 1). As expected, we found buildup choice-related signals in LIP neurons 138 under all cue conditions. As shown in PSTHs of the example cells (Figure 2a and Supplementary

139 Figure 2), there was generally an increasing divergence between the neuron's firing rate on trials 140 in which the monkey chose the target in the neuron's response field (IN choices, solid curves) and 141 trials in which the opposite target was chosen (OUT choices, dashed curves). Importantly, in all cue 142 conditions, the buildup choice signals tended to be stronger for heading directions more distant 143 away from straight ahead (Supplementary Figure 3), suggesting that the response of LIP neurons 144 reflects the accumulation of visual and vestibular sensory evidence for heading judgments.

146 To better quantify the choice-related signals, we used a ROC analysis to generate an index of choice 147 divergence (CD) $\left({ }^{23}\right.$ Raposo, et al., 2014) that measures the strength of the choice signals (Figure 148 2b). The four cells illustrated in Fig. 2 exhibited canonical ramping choice signals, but their CDs 149 varied greatly across cue conditions. For example, for Cell 1, the CD was largest in the combined 150 condition, modest in the visual condition, and smallest in the vestibular condition. By contrast, for 151 Cell 4, the CD was largest in the vestibular condition. The heterogeneity of choice signals was also 152 manifest at the population level. Approximately half of the LIP neurons exhibited statistically 153 significant $\mathrm{CD}(\mathrm{p}<0.05$, two-sided permutation test) in each cue condition (vestibular: 52\%, visual: $15446 \%$, combined: 59\%; Figure 2c), but these three subpopulations did not fully overlap. While more 155 than two thirds of neurons (76\%) had significant choice signals in any of the three 


\section{a}

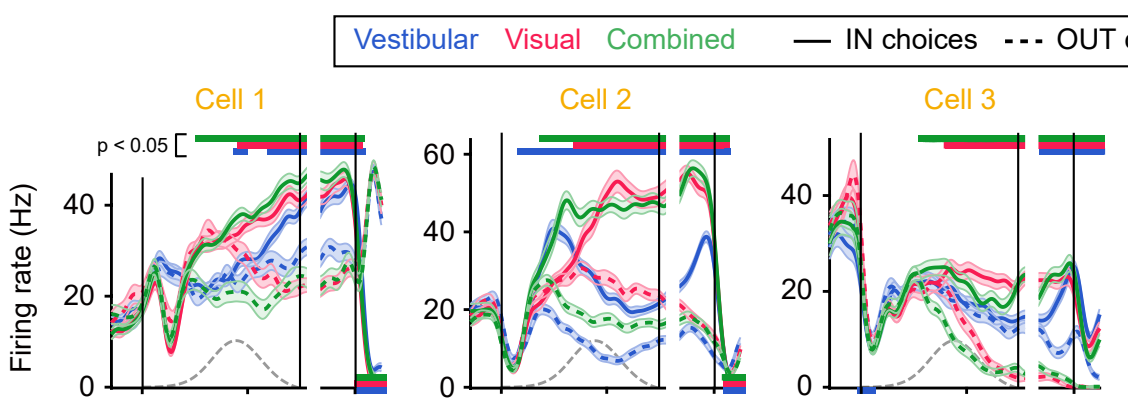

b
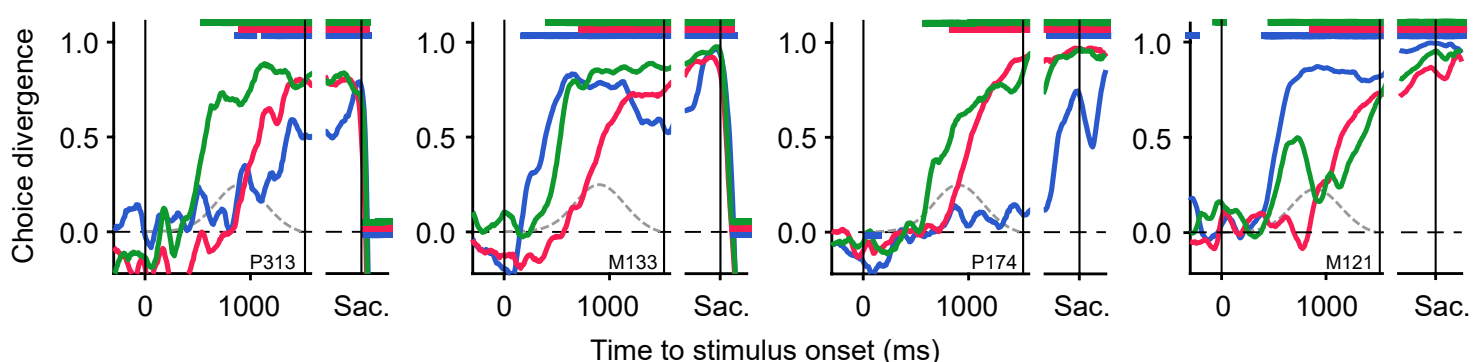

C

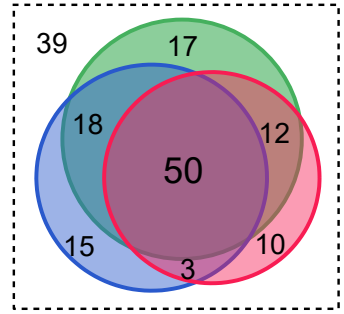

$\begin{array}{ll}\text { Total } & 164 \\ \text { Vestibular choice } & 86(52 \%) \\ \text { Visual choice } & 75(46 \%) \\ \text { Combined choice } & 97(59 \%) \\ \text { Any choice } & 125(76 \%) \\ \text { All choices } & 50(30 \%) \\ \text { None choice } & 39(24 \%)\end{array}$

Figure 2 Heterogeneous choice signals in LIP population.

(a) Peri-stimulus time histograms (PSTHs) of four example cells. Spike trains were aligned to stimulus onset (left subpanels) and saccade onset (right subpanels), respectively, and grouped by cue condition and monkey's choice. Vestibular, blue; visual, red; combined, green. Toward the cell's response field (RF), or IN choices, solid curves; away from the cell's RF, or OUT choices, dashed curves. Mean firing rates were computed from 10-ms time windows and smoothed with a Gaussian $(\sigma=50 \mathrm{~ms})$; only correct trials or $0^{\circ}$ heading trials were included. Shaded error bands, s.e.m. Horizontal color bars represent time epochs in which IN and OUT trials have significantly different firing rates $(p<0.05$, t-test), with the color indicating cue condition and the position indicating the relationship between IN and OUT firings (IN > OUT, top; IN < OUT, bottom). Gray dashed curves represent the actual speed profile measured by an accelerometer attached to the motion platform. (b) Choice divergence (CD) of the same four cells. CD ranged from -1 to 1 and was derived from ROC analysis for PSTHs in each 10-ms window (see Methods). Horizontal color bars are the same as in a except that $p$-values were from permutation test $(n=1000)$. (c) Venn diagram showing the distribution of choice signals. Numbers within colored areas indicate the numbers of neurons that have significant grand CDs (CD computed from all spikes in 0-1500 ms) under the corresponding combinations of cue conditions. 
157 conditions ("Any choice" cells in Figure 2c), only a third of neurons (30\%) had significant choice

158 signals in all of the three conditions ("All choices" cells in Figure 2c).

160 Apart from the heterogeneous choice signals, LIP also encodes heterogeneous sensory modality

161 signals. For example, Cell \#12 in Supplementary Figure 2 exhibited differentiated firing rates

162 across cue conditions without much choice-related signal. In fact, as shown in Supplementary

163 Figure 4a, the majority of LIP neurons actually carried mixed choice and modality signals,

164 exhibiting a category-free like neural representation as previously seen in rat posterior parietal

165 cortex $\left({ }^{23}\right.$ Raposo, et al., 2014). However, although randomly mixed at the single neuron level, the

166 choice and modality signals can still be linearly decoded from the LIP population (Supplementary

167 Figure 5). Therefore, we ignore the mixed modality signals thereafter, since they are irrelevant to

168 our heading discrimination task and orthogonal to the decision signals that we really care about.

170 Another potential difficulty in interpreting LIP activity arises from the fact that LIP neurons also 171 multiplex a combination of temporally overlapping decision- and non-decision- signals ( ${ }^{25} \mathbf{P a r k}$, $\boldsymbol{e t}$

172 al., 2014; ${ }^{26}$ Meister, et al., 2013). In particular, the signal of saccade preparation may interfere with

173 the one reflecting evidence accumulation $\left({ }^{14}\right.$ Shadlen and Newsome, 2001). However, this was not

174 likely to be an issue in our study. In our fixed-duration task, we introduced a 300-600 ms delay

175 between the stimulus offset and the time at which the monkey was allowed to saccade (see

176 Methods). Moreover, the monkeys tended to stop integrating evidence around $500 \mathrm{~ms}$ prior to the

177 stimulus offset (see Figure 3b and below), further separating in time the processes of evidence

178 accumulation and saccade preparation. Therefore, the premotor activity of LIP should not play a

179 significant role in our analysis of multisensory evidence accumulation.

180

181 LIP integrates vestibular acceleration and visual velocity 
182 Despite the high degree of heterogeneity, there was nonetheless a property shared amongst the LIP

183 neurons, namely, the temporal dynamics of the ramping activity was significantly faster in the

184 vestibular and combined conditions than in the visual condition (Figure 3). This was evident not

185 only in the averaged rate-based or ROC-based measures (“Any choice" cells, Figure 3a, b), but

186 also in the cell-by-cell analysis (Figure 3c). Notably, the averaged divergence time under the

187 vestibular and combined conditions aligned well to the acceleration peak of the Gaussian-shape

188 motion profile, whereas the divergence time under the visual condition better aligned to the velocity

189 peak (Figure 3c, dashed curves). This suggests that the physical quantities being integrated over

190 time are speed for the visual stimulus and acceleration for the vestibular stimulus.

192 An alternative explanation, however, might be that the apparent $\sim 400 \mathrm{~ms}$ interval between the

193 vestibular and visual ramping was caused purely by a difference in their sensory latencies rather

194 than in their underlying physical quantities. For example, LIP activity could have been driven by

195 an ultrafast vestibular signal but a slow visual signal, both of which followed the velocity of the

196 motion. To test this, we designed an experiment in which we used two distinct velocity profiles, a

197 wide one and a narrow one (Figure 3d). These profiles were designed to have temporally aligned

198 velocity peaks but misaligned acceleration peaks. If our original physical-quantity hypothesis was

199 correct, we would expect the visual ramping to remain nearly the same under both profiles, while

200 the vestibular ramping should start earlier for the wide profile than for the narrow one, thus

201 reflecting the earlier acceleration peak under the wide profile. In contrast, if the sensory-latency

202 hypothesis was correct, there should be no shift in either the vestibular or visual ramping across the

203 two profiles. Our data matches the first prediction (Figure 3e, f). In other words, the temporal

204 discrepancy between the vestibular and visual ramping activities indeed resulted from different

205 physical quantities underlying the momentary evidence fed into LIP. This physiological finding

206 echoed a recent psychophysical study showing that, at the behavioral level, human subjects

207 optimally integrate vestibular and visual momentary evidence with reliability following the 


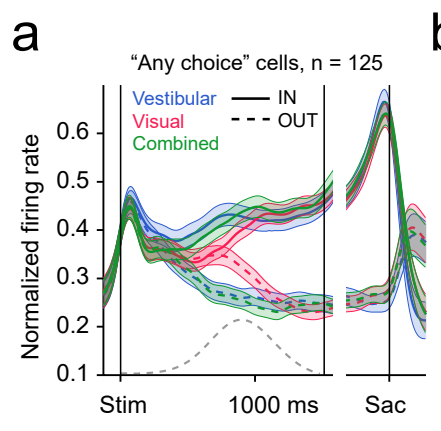

b

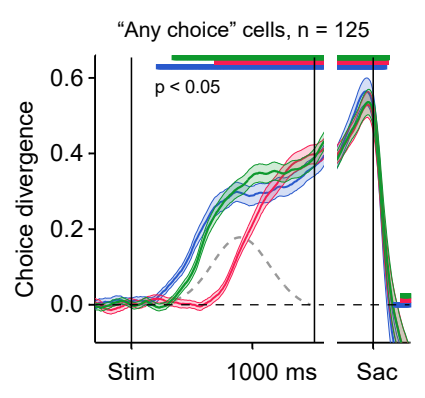

d

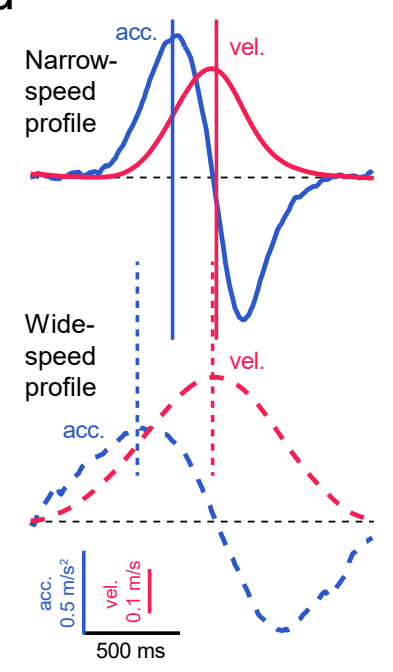

e

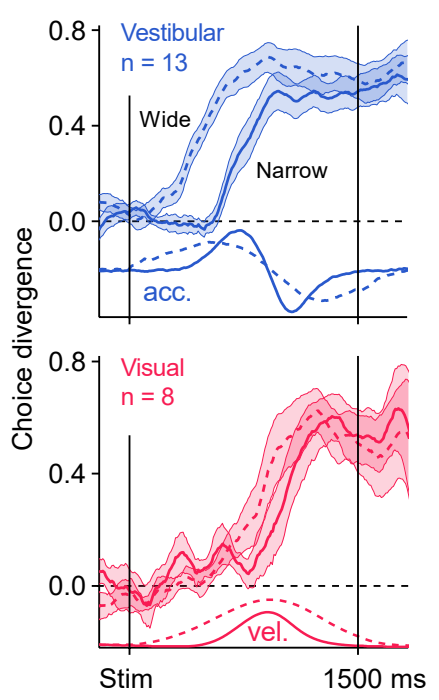

C

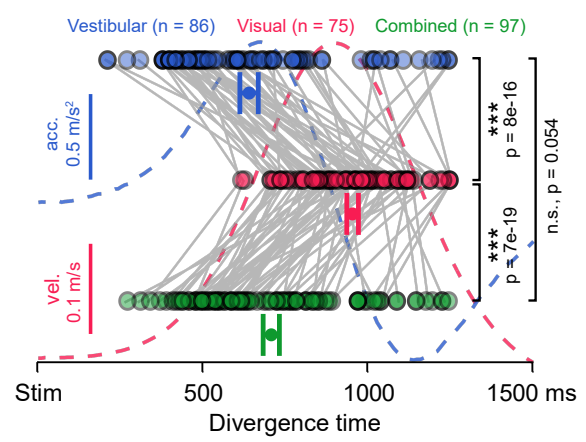

$f$

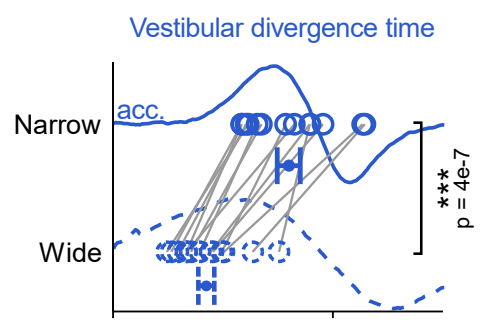

Visual divergence time

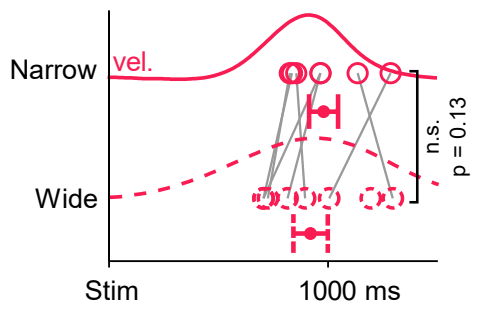

Figure 3 LIP integrates vestibular acceleration but visual speed.

(a and b) Population average of normalized PSTHs (a) and CD (b) from 125 "any choice" cells. The vestibular (blue) and combined (green) CDs ramped up much earlier than the visual one (red). Horizontal color bars indicate the time epochs in which population CDs are significantly larger than zero ( $p<0.05$, t-test). Gray dashed curve, the actual Gaussian speed profile; shaded error bands, s.e.m. (c) Divergence time of cells with significant grand CD for each condition. Divergence time was defined as the first occurrence of a $250-\mathrm{ms}$ window in which $C D$ was consistently larger than zero ( $p$ $<0.05$, permutation test). Gray lines connect data from the same cells; acceleration and speed profiles shown in the background. Data points with horizontal error bars, mean \pm s.e.m. of population divergence time; $p$ values, t-test. (d) Two motion profiles used to isolate contributions of acceleration and speed to LIP ramping. Top and solid, the narrow-speed profile; bottom and dashed, the widespeed profile; blue, acceleration; red, speed. Note that by widening the speed profile, we shifted the time of acceleration peak forward (blue vertical lines) while keeping the speed peak unchanged (red vertical lines). (e) Vestibular and visual CDs under the two motion profiles. (f) Comparison of divergence time between narrow and wide profiles. Note that the vestibular divergence time was significantly shifted, whereas the visual one was not, indicating that LIP integrates sensory evidence from vestibular acceleration and visual speed. 
amplitude of acceleration and velocity, respectively ( ${ }^{8}$ Drugowitsch, et al., 2014).

\section{Network model implementing ilPPC for multisensory decision making}

212 Next, we developed a neural model of multisensory decision making (refer to as M1 thereafter)

213 which takes as input vestibular neurons tuned to acceleration and visual neurons tuned to velocity

214 as observed in vivo (equation (2) and (3) in Methods; Figure 4a). These inputs converge onto an

215 integration layer which takes the sum of the visual and vestibular inputs, as well as integrates this

216 summed input over time. This layer projects in turn to an output layer, labeled LIP, which sums the

217 integrated visuo-vestibular inputs with the activity from another input layer encoding the two

218 possible targets to which the animal can eventually saccade (Figure $\mathbf{4 b}, \mathbf{c}$ ). As long as the input

219 layers encode the sensory inputs with ilPPC, this simple network can be shown analytically to

220 implement the Bayes optimal solution even when the reliability of the sensory inputs vary over

221 time as is the case in our experiment ( ${ }^{9}$ Beck, et al., 2008; ${ }^{10} \mathrm{Ma}$, et al., 2006). Note that separating

222 the integration layer from the LIP layer is not critical to our results. We did so to reflect the fact

223 that current experimental data suggest that LIP may not be the layer performing the integration per

224 se, but may only reflect the results of this integration $\left({ }^{27} \mathrm{Katz}\right.$, et al., 2016).

226 In an ilPPC, the gain, or amplitude, of the tuning curves of the neurons should be proportional to

227 the reliability of the encoded variable. For instance, in the case of vestibular neurons, the amplitude

228 of the tuning curves to heading should scale with acceleration. In vivo, however, the responses of

229 vestibular and visual neurons are not fully consistent with the assumption of ilPPC because while

230 the amplitude does increase with reliability, in some neurons, the baseline activity decreases with

231 reliability (equation (2) and (3) in Methods and Supplementary Figure 6a). This violation of the

232 ilPPC assumption implies that a simple sum of activity could incur an information loss. Fortunately,

233 this information loss is small for a population of neurons with tuning properties similar to what has 

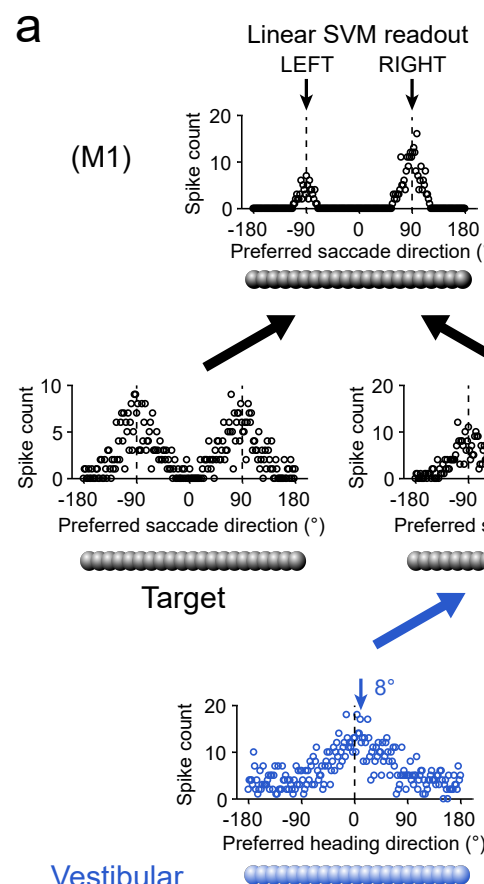

$M$ readout RIGHT

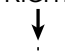

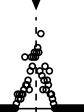

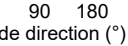

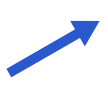
LP

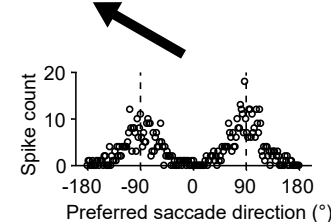
Preferred saccade direction $\left({ }^{\circ}\right)$ Integrator
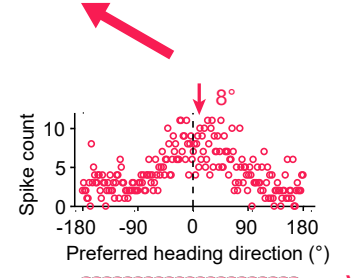

comenomomomonemo Visual

b

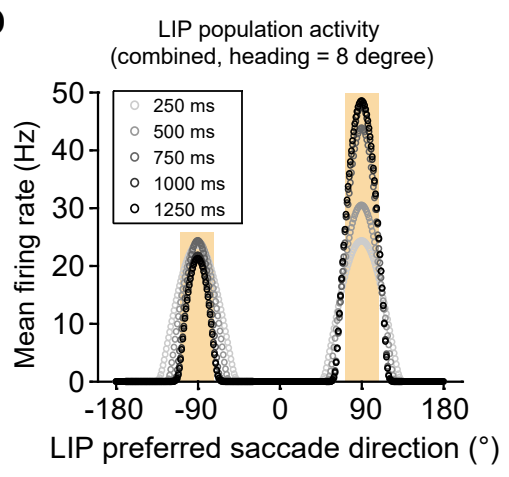

C

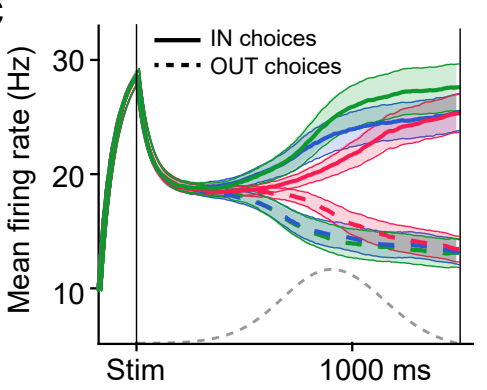

Figure 4 Neural network model with invariant linear probabilistic population codes (ilPPC).

(a) Network architecture of model M1. The model consists of three interconnected layers of linearnonlinear-Poisson units (inset). Units in Vestibular and Visual layers have bell-shape ilPPCcompatible tuning curves for heading direction and receive heading stimuli with temporal dynamics following acceleration and speed, respectively. The intermediate Integrator layer simply sums the incoming spikes from the two sensory layers over time and transforms the tuning curves for heading direction to that for saccade direction $\left(-90^{\circ}\right.$, leftward choice; $+90^{\circ}$, rightward choice). The LIP layer receives the integrated heading inputs from the Integrator layer, together with visual responses triggered by the two saccade targets. LIP units also have lateral connections implementing shortrange excitation and long-range inhibition. Once a decision boundary is hit, or when the end of the trial is reached (1.5 s), LIP activity is decoded by a linear support vector machine for action selection (see Methods). Circles indicate representative patterns of activity for each layer; spike counts from 800-1000 ms; combined condition, $8^{\circ}$ heading. (b) Population firing rate in the LIP layer at five different time points (the same stimulus as in a, averaged over 100 repetitions). (c) Average PSTHs across LIP population. Trials included three cue conditions and nine heading directions $\left( \pm 8^{\circ}, \pm 4^{\circ}, \pm 2^{\circ}\right.$, $\left.\pm 1^{\circ}, 0^{\circ}\right)$. To mimic the experimental procedure, only units with preferred saccade direction close to $\pm 90^{\circ}$ were used (with deviation less than $20^{\circ}$; yellow shaded area in $\mathbf{b}$ ). Notations are the same as in Figure 2a and Figure 3a. 
235 been reported experimentally and information limiting correlations ( ${ }^{28}$ Moreno-Bote, et al., 2014).

236 Indeed, we found numerically that the information loss was around 5\% over a wide range of

237 parameters values (Fano factor, mean correlation, baseline changes, and so on) (Supplementary

238 Modeling and Supplementary Figure 6).

239

240 Importantly, we also endowed the network with a stopping mechanism which terminates sensory

241 integration whenever a function of the LIP population activity reaches a preset threshold (see

242 Methods). Our experiment is not a reaction time experiment and may not require, in principle, such

243 a stopping bound. However, as can be seen in Figure 3b, LIP population response saturates around

244 1s, suggesting that evidence integration stops prematurely. This is indeed consistent with the

245 previous results suggesting that animals and humans use a stopping bound even in fixed duration

246 experiments $\left({ }^{29}\right.$ Kiani, et al., 2008).

248 LIP data are compatible with the ilPPC framework

249 In the first set of simulations on M1, we adjusted the height of the stopping bounds and found that

250 the model can replicate the near optimal animals' performance (Figure 5a). We then plotted the 251 activity of a typical output neuron (in the LIP layer) in all three conditions. As expected, the activity 252 in the combined condition is roughly equal to the sum of the vestibular-only activity and visual253 only activities (Figure 5b), at least in the first half of the trial. In the second half of the trial, the 254 activity in the combined condition deviates strongly from the sum because the traces correspond to 255 averages across trials that terminated at different times on different trials due to the stopping bound.

257 Neurons in M1 are homogeneous in the sense that they all take a perfect sum of their vestibular and 258 visual inputs. Importantly, however, optimal integration does not require such a perfect sum; it can 259 also be achieved with random linear combinations of vestibular and visual inputs 

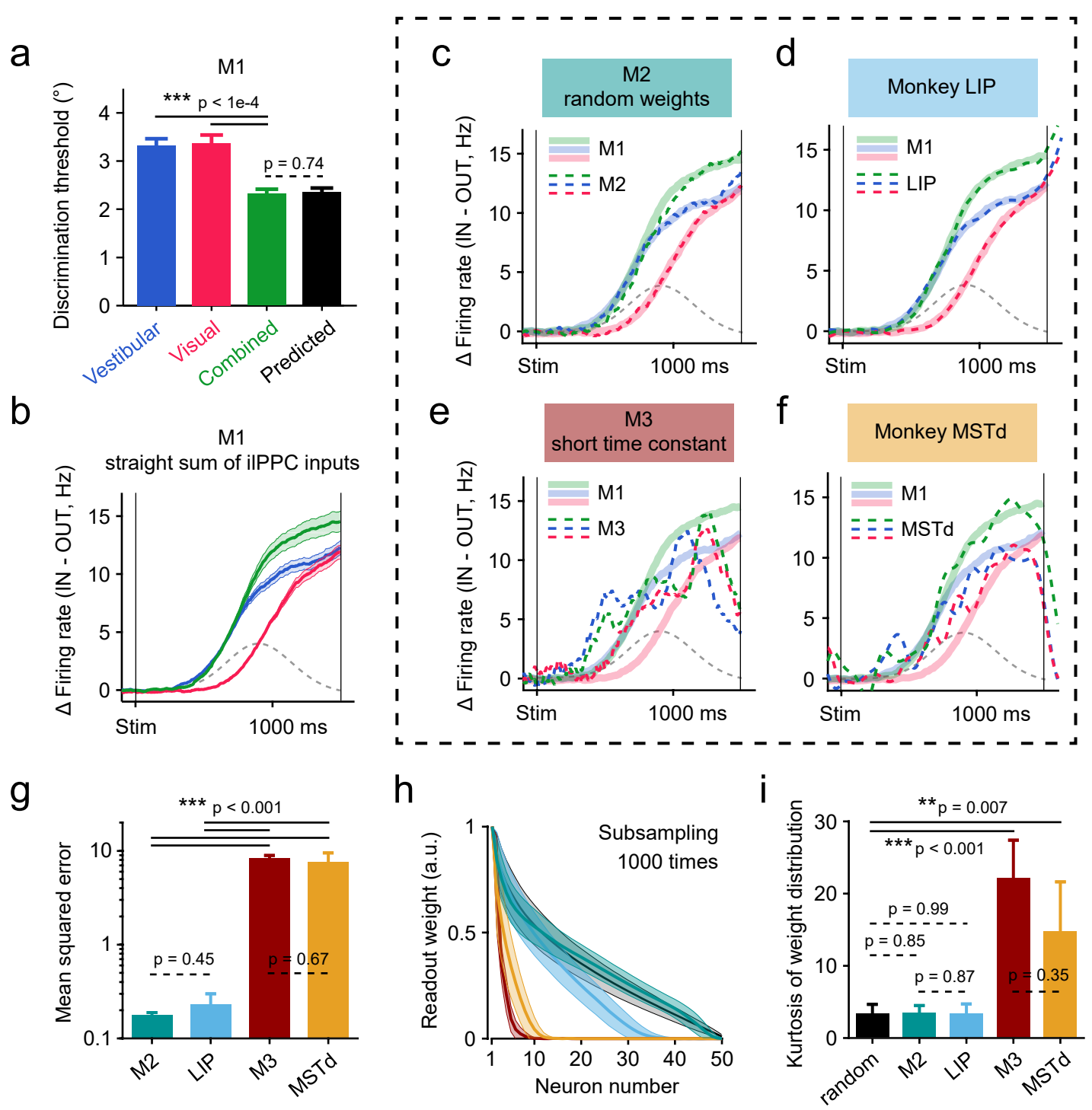

Figure 5 Optimal ilPPC model M1 can be linearly approximated by M2 and LIP but not by M3 and MSTd.

(a) Model M1 exhibited near-optimal behavior as the monkey. The psychophysical threshold under the combined condition (green) was indistinguishable from the Bayesian optimal prediction (black). (b) Ramping activity of M1 computed as the difference of PSTHs for IN and OUT trials. Activities from hypothetical units in the LIP layer with preferred direction close to $\pm 90^{\circ}$ were averaged together (see Figure 4c and Methods). Since M1 is optimal and homogeneous, we refer to M1's activities as "optimal traces" (see the main text). Notations are the same as before. (c) Optimal traces from M1 (thick shaded bands) can be linearly reconstructed by population activities obtained from a heterogenous model M2 (dashed curves). Model M2 had the same network architecture as M1 except that it relies on random combinations of ilPPC inputs in the integration layer (see Methods). (d) Optimal traces can also be linearly reconstructed by heterogenous single neuron activities from the LIP data. The similarity between $\mathbf{c}$ and $\mathbf{d}$ suggests that both model M2 and monkey LIP are heterogeneous variations of to the optimal ilPPC model M1. (e and f) In contrast, the optimal traces cannot be reconstructed from activities of a suboptimal model M3 (e) or from the MSTd data (f), presumably because the time constants in M3 and MSTd were too short. (g) Mean squared error of the fits in panels $\mathbf{c}-\mathbf{f}$. Error bars and $p$ values were from subsampling test ( $n=50$ neurons, 1000 times). (h) Normalized readout weights ordered by magnitude. Shaded error bands indicate standard deviations of the subsampling distributions. (i) The kurtosis of the distributions of weights. The black curve in (h) and black bar in (e) were from random readout weights (see Methods). 
261 ( ${ }^{10} \mathrm{Ma}$, et al., 2006). Accordingly, we simulated a second model, refer to as M2, in which the visual

262 and vestibular weights of each neuron were drawn from lognormal distributions (Figure 5c and see

263 Methods). Like M1, model M2 can be tuned to reproduce the Bayes optimal discrimination

264 thresholds (Supplementary Figure 7a, b). However, in contrast to model M1, the neurons showed

265 a wide range of response profiles closer to what we observed in vivo (Supplementary Figure 7c).

266 In particular, we found that the distribution of visual and vestibular weights was similar in the

267 model and in LIP data (Supplementary Figure 7d).

269 Since model M2 is a linear combination away from model M1, we tested whether the response of

270 M1 neurons could be estimated by linearly combining the response of M2 neurons. Multivariate

271 linear regression confirmed that M1 response profiles could indeed be perfectly reproduced by

272 linearly combining M2 responses (Figure 5c). Since LIP neurons also appear to be computing

273 random linear combinations of visual and vestibular inputs, the same result should hold for LIP

274 responses. This is indeed what we found: the response of M1 neurons can be closely approximated

275 by linearly combining the response of LIP neurons (Figure 5d, g and Supplementary Figure 9).

277 This last result is key: it suggests that LIP neurons behave quite similarly to the neurons in M2. The

278 two sets of neurons, however, differ quite significantly in how they integrate their inputs over time.

279 LIP neurons display a wide variety of temporal profiles (see Supplementary Figure 2), suggesting

280 that very few neurons act like perfect temporal integrators, in contrast to M2 neurons. Nonetheless,

281 the fact that linear combinations of LIP neurons could reproduce the response of M1 neurons

282 indicates that LIP responses provide a basis set sufficiently varied to allow perfect integration at

283 the population level, a result consistent with what has been recently reported in the posterior parietal

284 cortex of rats engaged in a perceptual decision making task ( ${ }^{30}$ Scott, et al., 2017).

286 In addition to this second model, we simulated a third model (M3) in which the time constant of 
287 the integration layer was reduced to $100 \mathrm{~ms}$. Interestingly, we found that it was not possible to

288 linearly combine the responses of M3 output neurons to reproduce the traces of the optimal model

289 M1 (Figure 5e, g), thus emphasizing the importance of long integration time constant for fitting

290 the optimal model. We also wondered whether M1 could be fitted by the response of MSTd neurons,

291 which are known to combine visual and vestibular responses and whose time constant are believed

292 to be of the same order as model M3. We found that the fit to M1 from MSTd neurons was markedly

293 worse than those obtained from M2 and LIP but was close to that from M3 (Figure 5f, g). Moreover,

294 only a small fraction of cells contributed significantly to this fit, in sharp contrast to what we

295 observed in M2 and LIP (Figure 5h, i). In fact, the late phase of M1 responses was captured mostly

296 by MSTd cells with short time constants who seemed sensitive to deceleration, rather than

297 integrating cells (Supplementary Figure 8).

299 Finally, we computed the shuffled Fisher information over time for the models and the experimental

300 data (Figure 6). The Fisher information in a neuronal population is a measure inversely

301 proportional to the square of the discrimination threshold of an ideal observer $\left({ }^{31}\right.$ Beck, et al., 2011;

$302{ }^{32}$ Seung and Sompolinsky, 1993). The shuffled Fisher information is a related measure

303 corresponding to the information in a data set in which neurons are recorded one at a time, as

304 opposed to simultaneously, which is the case for our data set ( ${ }^{33}$ Series, et al., 2004) (see Methods).

305 Our network simulations revealed that the shuffled Fisher information should increase over time in

306 all conditions, reflecting the temporal accumulation of evidence (Figure 6a). In addition, we

307 observed that this rise in information starts earlier in the vestibular condition than in the visual one

308 because of the temporal offset between acceleration and velocity. In the combined condition, the

309 Fisher information follows at first the vestibular condition before exceeding the vestibular trace

310 once the visual information becomes available. Remarkably, the shuffled Fisher information

311 estimated from the LIP responses follows qualitatively the same trend as the ones observed in the

312 model (Figure 6b). In contrast to M2 and LIP neurons, shuffled Fisher information in M3 and 

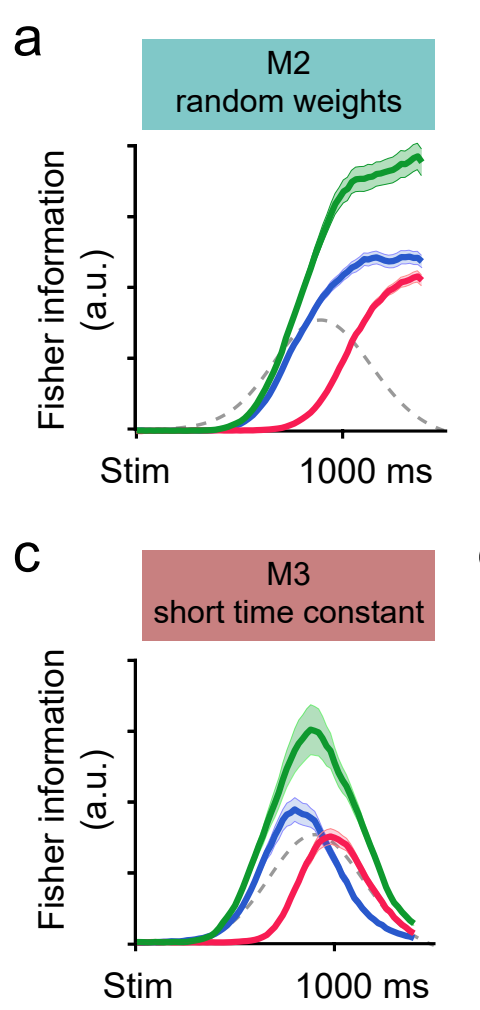

b

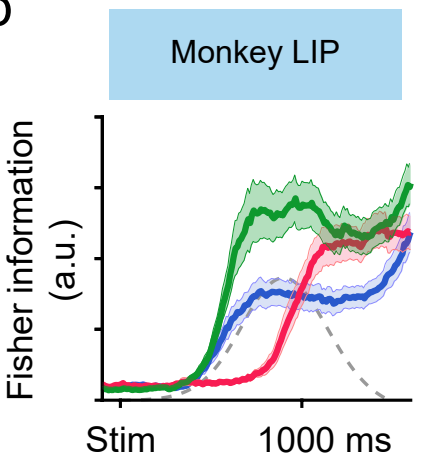

d

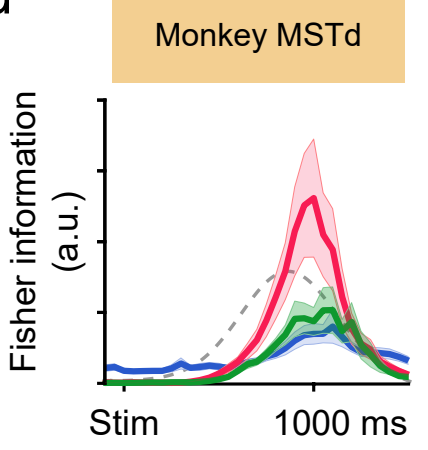

Figure 6 Shuffled Fisher information for the model and the experimental data.

(a) Shuffled Fisher information of M2 calculated by $I_{\text {shuffled }}=\sum_{i} f_{i}^{\prime 2} / \sigma_{\mathrm{i}}^{2}$, where $f_{i}{ }^{\prime}$ denotes the derivative of the local tuning curve of the $i$ th neuron and $\sigma_{i}^{2}$ denotes the averaged variance of its responses around $0^{\circ}$ (see Methods). Both correct and wrong trials were included. Shaded error bands, s.e.m. estimated from bootstrap. Note that the absolute value of shuffled Fisher information is arbitrary. (b-d) Same as in a but for the monkey LIP data, the M3 responses, and the monkey MSTd data, respectively. Note that LIP is similar to M2, and MSTd to M3. 
314 MSTd followed the profile expected for neurons with short time constant: it simply reflected the 315 velocity profile of the stimulus and did not exhibit the plateau expected from a decision area 316 (Figure 6c, d).

317

318 Taken together, our results are consistent with the notion that MSTd neurons provide the visual 319 momentary evidence for decision making, while LIP circuits, or circuits upstream from LIP, 320 implement the near optimal solution of model M1, in the sense that the LIP population activity is a 321 mere linear transformation away from that solution. 


\section{Discussion}

323 Integrating ever-changing sensory inputs from different sources across time is crucial for animals

324 to optimize their decisions in a complex environment, yet little is known about the underlying 325 mechanisms, either experimentally or theoretically. In the current study, we present, to the best of 326 our knowledge, the first electrophysiological data on multisensory decision making from non327 human primates. We found that LIP neurons in the macaque posterior parietal cortex encode 328 ramping decision signals not only for the visual condition, as widely shown in the literature, but 329 also for the vestibular and combined conditions, except with distinct temporal dynamics.

330 Importantly, these data are compatible with an ilPPC framework where optimal multisensory 331 evidence accumulation is achieved by simply summing sensory inputs across both modalities and 332 time, even with mismatched temporal profiles of cue reliabilities and with heterogeneous sensory333 motor representation. Therefore, our results provide the first neural correlate of optimal 334 multisensory decision making.

336 Distinct visual and vestibular temporal dynamics in LIP

337 By comparing the temporal dynamics of LIP population under different modalities, we found that

338 LIP neurons accumulate vestibular acceleration and visual speed, which serve as momentary 339 evidence for their respective modalities. These findings may seem confusing at first glance, since 340 it is more intuitive to assume that neural circuits would combine evidence with the same temporal 341 dynamics across cues, namely, either visual and vestibular speed or visual and vestibular 342 acceleration $\left({ }^{19} \mathrm{Gu}\right.$, et al., 2006; ${ }^{34} \mathrm{Chen}$, et al., 2011a; ${ }^{35}$ Fetsch, et al., 2010; ${ }^{36} \mathrm{Smith}$, et al., 2017).

343 In support of this idea, recent studies have found a remarkable transformation from acceleration-

344 dominated to speed-dominated vestibular signal along the vestibular pathway, i.e. from peripheral

345 otolith organs to the central nervous system ( ${ }^{37}$ Laurens, et al., 2017), as well as a moderate but 346 noticeable further transformation along several sensory cortices $\left({ }^{19} \mathrm{Gu}\right.$, et al., 2006 ; ${ }^{34} \mathrm{Chen}$, et al., 
2011a; ${ }^{35}$ Fetsch, et al., 2010; ${ }^{37}$ Laurens, et al., 2017). Given that visual motion responses are

348 typically dominated by speed $\left({ }^{19} \mathrm{Gu}\right.$, et al., 2006; ${ }^{38}$ Lisberger and Movshon, 1999), one would

349 think that the brain may deliberately turn the vestibular signal from acceleration- to speed-sensitive

350 to facilitate the combination with the visual signal.

352 However, if the vestibular momentary evidence is proportional to acceleration corrupted by white

353 noise across time, integrating this evidence to obtain a velocity signal would not simplify decision

354 making. On the contrary, this step would introduce temporal correlations $\left({ }^{39}\right.$ Churchland, et al.,

355 2011), in which case, even with ilPPC, a simple sum of the momentary evidence would no longer

356 be optimal ( ${ }^{6}$ Bogacz, et al., 2006). Instead, downstream circuits would have to compute a weighted

357 sum of the sensory evidence, which would effectively differentiate the momentary evidence before

358 summing them. In other words, optimal integration would effectively recover the original

359 acceleration signals. Our results, along with previous psychophysical results $\left({ }^{8}\right.$ Drugowitsch, et al.,

360 2014), strongly suggest that the brain does not go through this extra step and uses the acceleration

361 signals as momentary evidence instead.

363 Multisensory convergence in the brain for heading decision

364 One of the long-standing questions about multisensory integration is whether integration takes

365 place early or late along the sensory streams $\left({ }^{40}\right.$ Bizley, et al., 2016). There are clear signs of 366 multisensory responses in relatively early- or mid- stage of sensory areas, thus supporting the early

367 theory $\left({ }^{41} \mathbf{G u}, \mathbf{2 0 1 8}\right)$. Our results are more consistent with the late-convergence theory in which

368 multisensory momentary evidence are combined across modalities and time in decision areas such

369 as LIP. However, this dichotomy between early and late theories does not necessarily make sense

370 given the recurrent nature of the cortical circuitry. In a highly recurrent network, it is notoriously

371 difficult to identify a node as a primary site of integration. Thus, integration might take place 
372 simultaneously across multiple sites but in such a way that the output of the computation is

373 consistent across sites. For example, Deneve, et al. ${ }^{42}$ demonstrated how this could take place in a

374 large recurrent network performing optimal multisensory integration, though their work did not

375 consider the problem of temporal integration.

377 It might be possible to gain further insight into the distributed nature of multisensory decision

378 making by combining the previous models with the one we have presented here. Such an extended

379 model might explain why vestibular momentary evidence is tuned to velocity by the time they

380 appear in MSTd $\left({ }^{37}\right.$ Laurens, et al., 2017; $\left.{ }^{41} \mathbf{G u}, \mathbf{2 0 1 8}\right)$, and why this velocity tuned vestibular input

381 does not appear to be integrated in LIP. It could also shed light on recent physiological experiments

382 in which electrical microstimulation and chemical inactivation in MSTd could dramatically affect

383 heading discrimination based on optic flow while this effect was largely negligible in the vestibular

384 condition $\left({ }^{\mathbf{4}} \mathbf{G u}\right.$, et al., 2012). By contrast, and in accord with our finding that LIP integrates

385 vestibular acceleration, inactivating the vestibular cortex PIVC, where vestibular momentary

386 evidence is dominated by acceleration $\left({ }^{34}\right.$ Chen, et al., 2011a; ${ }^{37}$ Laurens, et al., 2017), substantially

387 diminished the macaque's heading ability based on vestibular cue $\left({ }^{44} \mathbf{C h e n}\right.$, et al., 2016). Note,

388 however, a detailed construction of such a model lies beyond the scope of the present study but will

389 eventually be required for a multi-area theory of multisensory decision making.

\section{Computational models for multisensory decision making}

392 Our results indicate that, at the population level, LIP implements an optimal solution for 393 multisensory decision making under the assumption that the sensory inputs are encoded with ilPPC.

394 This assumption is not perfectly satisfied in our experiment since the visual and vestibular inputs 395 deviate from pure ilPPCs, but we saw that this deviation introduces only a minor information loss.

396 While these results provide the first experimental support for the ilPPC theory of multisensory 
397 decision making, it will be important to test in future experiments other predictions of this

398 framework. In particular, the ilPPC theory predicts that LIP activity encodes a full probability

399 distribution over choices given the evidence so far ( ${ }^{9}$ Beck, et al., 2008). Testing this prediction

400 thoroughly requires simultaneous recording of LIP ensemble, manipulating the cue reliability

401 (motion profile or visual coherence) on a trial-by-trial basis, and preferably engaging the animals

402 in a reaction-time task, all of which should be addressed in future studies.

404 There are of course other models of decision making which could potentially account for the 405 responses we have observed in LIP $\left({ }^{45}\right.$ Chandrasekaran, 2017). In particular, it has been argued 406 that LIP is part of a network of areas implementing point attractor networks $\left({ }^{46} \mathbf{W o n g}\right.$ and Wang, 407 2006; ${ }^{47}$ Wang, 2002). However, it is not immediately clear how this approach can be generalized 408 to the type of decision we have considered here. Indeed, as we have seen, the optimal solution 409 depends critically on the code that is used to encode the momentary evidence. To the extent that 410 this code is close to an ilPPC, the optimal solution is to sum the inputs spikes, in which case one 411 needs a line attractor network, which is effectively what our network approximates. Therefore, as 412 long as these previous models of decision making are fine-tuned to approximate line attractor 413 networks, and as long as they are fed ilPPCs as inputs, the two classes of models would be 414 equivalent.

416 Training recurrent neural network (RNNs) on our task $\left({ }^{48}\right.$ Mante, et al., 2013; ${ }^{49}$ Song, et al., 2017) 417 provides a third alternative for modeling multisensory decision making. We also tried this approach 418 and found that the resulting network was capable of reproducing the behavioral thresholds of the 419 animal while exhibiting a wide variety of single neuron responses similar to what we saw in LIP (

420 Supplementary Figure 10). Nonetheless, this approach has one major drawback: it makes it very 421 difficult to understand how the network solves the task. We could try to reverse engineer the 422 network, but given that an analytical solution can be derived from first principles for our task, and 

not certified by peer review) is the author/funder. All rights reserved. No reuse allowed without permission.

423 given that this solution is close to what we observed in LIP, it is unclear what insight could be

424 gained from the recurrent network. In contrast, our ilPPC model provides a close approximation to

425 the optimal solution, consistent with the experimental results, along with a clear understanding as

426 to why this approach is optimal.

427

428 


\section{Methods}

\section{$430 \quad$ Subjects and Apparatus}

431 All animal procedures were approved by the Animal Care Committee of Shanghai Institutes for

432 Biological Sciences, Chinese Academy of Sciences and have been described previously in detail

433 ( ${ }^{12} \mathbf{G u}$, et al., 2008; ${ }^{19} \mathbf{G u}$, et al., 2006). Briefly, two male adult rhesus monkeys, Monkey P and

434 Monkey M, weighing $\sim 8 \mathrm{~kg}$, were chronically implanted with a lightweight plastic ring for head

435 restraint and a scleral coil for monitoring eye movements (Riverbend Instruments). During

436 experiments, the monkey sat comfortably in a primate chair mounted on top of a custom-built

437 virtual reality system, which consisted of a motion platform (MOOG MB-E-6DOF/12/1000KG)

438 and an LCD screen ( $\sim 30 \mathrm{~cm}$ of view distance and $\sim 90^{\circ} \times 90^{\circ}$ of visual angle; HP LD4201),

439 presenting vestibular and visual motion stimuli to the monkey, respectively. The stimuli were

440 controlled by customized $\mathrm{C}++$ software and synchronized with the electrophysiological recording

441 system by TEMPO (Reflective Computing, U.S.A).

443 To tune the synchronization between vestibular and visual stimuli, we rendered a virtual world-

444 fixed crosshair on the screen while projected a second crosshair at the same place on the screen

445 using a real world-fixed laser pen. When the platform was moving, we carefully adjusted a delay

446 parameter in the $\mathrm{C}++$ software (with $1 \mathrm{~ms}$ resolution) until the two crosshairs aligned precisely

447 together all the time, as verified by a high-speed camera (Meizu Pro 5) and/or a pair of back-to-

448 back mounted photodiodes. This synchronization procedure was repeated occasionally over the

449 whole period of data collection.

\section{$451 \quad$ Behavioral Tasks}

\section{$452 \quad$ Memory-guided Saccade Task}

453 We used the standard memory-guided saccade task ( ${ }^{50}$ Barash, et al., 1991) to characterize and 
454 select LIP cells for recording in the main decision-making experiments. The monkey fixated at a 455 central fixation point for $100 \mathrm{~ms}$ and then a saccade target flashed briefly (500 ms) in the periphery.

456 The monkey was required to maintain fixation during the delay period (1000 ms) until the fixation

457 point extinguished and then saccade to the remembered target location within $1000 \mathrm{~ms}$ for a liquid

458 reward. For all tasks in the present study, at any time when there existed a fixation point, trials were 459 aborted immediately if the monkey's gaze deviated from a $2^{\circ} \times 2^{\circ}$ electronic window around the 460 fixation point.

\section{Multisensory Heading Discrimination Task}

463 In the main experiments, we trained the monkeys to report their direction of self-motion in a two464 alternative forced-choice heading discrimination task $\left({ }^{12} \mathbf{G u}\right.$, et al., 2008) (Figure 1). The monkey 465 initiated a trial by fixating on a central, head-fixed fixation point, and two choice targets then 466 appeared. The locations of the two targets were determined case-by-case for each recording session 467 (see below). After fixating for a short delay (100 ms), the monkey then began to experience a fixed468 duration $(1.5 \mathrm{~s})$ forward motion in the horizontal plane with a small leftward or rightward 469 component relative to straight ahead. The animals were required to maintain fixation during the 470 presentation of the motion stimuli. At the end of the trial, the motion ended, and the monkey was 471 required to maintain fixation for another 300-600 ms random delay (uniformly distributed) until 472 the fixation point disappeared, at which point the monkey was allowed to make a saccade choice 473 toward one of the two targets to report his perceived heading direction (left or right).

475 Across trials, nine heading angles $\left( \pm 8^{\circ}, \pm 4^{\circ}, \pm 2^{\circ}, \pm 1^{\circ}\right.$, and $\left.0^{\circ}\right)$ and three cue conditions (vestibular, 476 visual, and combined) were jointly interleaved, resulting in 27 unique stimulus conditions, each of 477 which was repeated $15 \pm 3$ (median \pm m.a.d.) times per one session. In a vestibular or a visual trial, 478 heading information was solely provided by inertial motion (real movement of the motion platform) 
481 the behavioral benefit of cue integration, we balanced the monkey's performance under the

482 vestibular and the visual conditions by manipulating the motion coherence of the optic flow (the 483 percentage of dots that moved coherently). The visual coherence was $12 \%$ and $8 \%$ for monkey $\mathrm{P}$ 484 and $\mathrm{M}$, respectively.

486 To ensure that the reliabilities of sensory cues varied throughout each trial, we used Gaussian-shape, 487 rather than constant, velocity profiles for all motion stimuli. In the main experiments, the Gaussian 488 profile had a displacement $d=0.2 \mathrm{~m}$ and a standard deviation $\sigma=210 \mathrm{~ms}$ (half duration at about $48960 \%$ of the peak velocity), resulting in a peak velocity $v_{\max }=0.37 \mathrm{~m} / \mathrm{s}$ and a peak acceleration $490 a_{\max }=1.1 \mathrm{~m} / \mathrm{s}^{2}$. In the experiment where we sought to independently vary the peak times of 491 velocity and acceleration (Figure 3), two additional sets of motion parameters were used. For the 492 narrow-speed profile, $d=0.10 \mathrm{~m}, \sigma=150 \mathrm{~ms}, v_{\max }=0.37 \mathrm{~m} / \mathrm{s}$, and $a_{\max }=1.1 \mathrm{~m} / \mathrm{s}^{2}$; for 493 the wide-speed profile, $d=0.25 \mathrm{~m}, \sigma=330 \mathrm{~ms}, v_{\max }=0.31 \mathrm{~m} / \mathrm{s}$, and $a_{\max }=0.6 \mathrm{~m} / \mathrm{s}^{2}$.

\section{Electrophysiology}

496 We carried out extracellular single-unit recordings as described previously $\left({ }^{12} \mathbf{G u}\right.$, et al., 2008) from 497 four hemispheres in two monkeys. For each hemisphere, reliable area mapping was first achieved 498 through cross-validation between structural MRI data and electrophysiological properties, 499 including transition patterns of gray/white matter along each penetration, sizes of visual 500 receptive/response field, strengths of spatial tuning to visual and vestibular heading stimuli, and 501 activities in the memory-guided saccade task. Based on the mapping results, Area LIP was 502 registered by its spatial relationships with other adjacent areas (VIP, Area 5, MSTd, etc.), its weak 503 sensory encoding of heading information, and its overall strong saccade-related activity 504 (Supplementary Figure 1). Our recording sites located in the ventral division of LIP, extending 
505 from $7-13 \mathrm{~mm}$ lateral to the midline and $-5 \mathrm{~mm}$ (posterior) to $+3 \mathrm{~mm}$ (anterior) relative to the

506 interaural plane.

508 Once we encountered a well-isolated single unit in LIP, we first explored its response field (RF) by

509 hand (using a flashing patch) and then examined its electrophysiological properties using the

510 memory-guided saccade task. The saccade target in each trial was randomly positioned at one of

511 the 8 locations $45^{\circ}$ apart on a circle centered on the fixation point $\left(5^{\circ}-25^{\circ}\right.$ radius, optimized

512 according to the cell's RF location). We calculated online the memory-saccade spatial tuning for

513 three response epochs: (1) visual response period, 75-400 ms from target onset; (2) delay period,

514 25-900 ms from target offset; and (3) presaccadic period, 200-50 ms before the saccade onset

515 (Supplementary Figure 2). The cell's spatio-temporal tunings were used to refine its RF location

516 (via vector sum) and to determine its inclusion in the subsequent decision-making task. Since the

517 decision-related activity of LIP neurons cannot be strongly predicted by the persistent activity

518 during the delay period alone ( ${ }^{26}$ Meister, et al., 2013) (Supplementary Figure 4b), we adopted a

519 wider cell selection criterion than conventionally used, in which we included cells that have

520 significant spatial selectivity for any of the three response epochs $\left({ }^{\mathbf{2 6}}\right.$ Meister, et al., 2013) (one-

521 way ANOVA, $\mathrm{p}<0.05,3-5$ repetitions). If the cell met this criterion, then we recorded its decision-

522 related activity while engaging the monkey in the main multisensory decision-making task, with

523 the two choice targets being positioned in its RF and $180^{\circ}$ opposite to its RF, respectively.

525 Although we collected data from a relatively broad sample of LIP neurons, we nevertheless had

526 two sampling biases during this process. First, we were biased toward cells with strong persistent

527 activity so that our multisensory data could be better compared with previous unisensory data in

528 the decision-making literature, where in most cases only these cells were recorded. Second, we

529 were biased toward cells with RF close to the horizontal line through the fixation point. Unlike the

530 classical random dot stimuli whose motion direction on the fronto-parallel plane can be aligned 
531 with the cell's RF (and the choice targets) session by session, our self-motion stimuli were always

532 on the horizontal plane and thus were not adjustable according to the cell's RF on the fronto-parallel

533 plane. As a result, the subjects had to make an additional mapping from their perceived heading

534 directions (always left or right) to the choice targets (often inclined, and in extreme cases, up or

535 down). Therefore, to make the task more intuitive to the monkeys and to minimize the potential

536 influence of this mapping step on neural activity, we discarded a cell if the angle between the

537 horizontal line and the line connecting the fixation point to its RF exceeded $60^{\circ}$, although we

538 observed little change in monkeys' behavior even when this angle approached $80^{\circ}$.

Data Analysis

Psychophysics

542 To quantify the behavioral performance for both the monkeys and the model in the multisensory

543 decision-making task, we constructed psychometric curves by plotting the proportion of "rightward"

544 choices as a function of heading (Figure 1c) and fitted them with cumulative Gaussian functions

545 ( ${ }^{12} \mathbf{G u}$, et al., 2008). The psychophysical threshold for each cue condition was defined as the

546 standard deviation of their respective Gaussian fit. The Bayesian optimal prediction of

547 psychophysical threshold under the combined condition $\sigma_{\text {prediction }}$ was solved from the inverse

548 variance rule $\left({ }^{24}\right.$ Knill and Richards, 1996)

$$
\frac{1}{\sigma_{\text {prediction }}^{2}}=\frac{1}{\sigma_{\text {vestibular }}^{2}}+\frac{1}{\sigma_{\text {visual }}^{2}}
$$

550 where $\sigma_{\text {vestibular }}$ and $\sigma_{\text {visual }}$ represent psychophysical thresholds under the vestibular and visual 551 conditions, respectively.

\section{Choice-related neural activities}

554 We constructed peri-stimulus time histograms (PSTHs) for two epochs of interest in a trial, the 
555 decision formation epoch and the saccade epoch, by aligning raw spike trains to the stimulus onset

556 and the saccade onset, respectively. Firing rates were computed in non-overlapping 10-ms bins and

557 smoothed over time by convolving with a Gaussian kernel $(\sigma=50 \mathrm{~ms})$. Unless otherwise noted,

558 only correct trials were used in the following analyses, except for the ambiguous $0^{\circ}$ heading where

559 we included all complete trials.

561 To illustrate the choice-related activity of a cell, we grouped the trials according to the monkey's

562 choice, i.e., trials ending up with a saccade toward the cell's RF (IN trials) versus trials ending up

563 with a saccade away from the cell's RF (OUT trials), and computed the averaged PSTHs of these

564 two groups of trials for each cue condition (Figure 2a). When averaged across cells, each cell's

565 PSTHs were normalized such that the cell's overall firing rate had a dynamic range of [0, 1] (Figure

566 3). To quantify the strength of choice signals and better visualize ramping activities, we calculated

567 choice divergence $\left({ }^{23}\right.$ Raposo, et al., 2014) for each 10 -ms time bin and for each cue condition using

568 receiver operating curve (ROC) analysis (Figure $\mathbf{2 b}$ ). Choice divergence ranged from -1 to 1 and

569 was defined as $2 \times(\mathrm{AUC}-0.5)$, where AUC represents the area under the ROC curve derived

570 from PSTHs of IN and OUT trials. To capture the onset of choice signals, we computed a

571 divergence time defined as the time of the first occurrence of a 250-ms window (25 successive 10-

572 ms bins) in which choice divergence was consistently and significantly larger than 0 (Figure 3c,

573 f). The statistical significance of choice divergence $(p<0.05$, relative to the chance level of 0$)$ was

574 assessed by two-sided permutation test (1000 permutations). We also calculated a grand choice

575 divergence which ignored temporal information and used all the spikes in the decision formation

576 epoch (0-1500 ms from the stimulus onset). The same permutation test was performed on the grand

577 choice divergence to determine whether a cell had overall significant choice signals for a certain

578 cue condition (for example, in Figure 2c). 


\section{Linear Fitting of Mean Firing Rates}

581 We fitted a linear weighted summation model to predict neural responses under the combined 582 condition with those under the single cue conditions, using $\left({ }^{12} \mathbf{G u}\right.$, et al., 2008)

$$
r_{\text {combined }}=w_{\text {vestibular }} r_{\text {vestibular }}+w_{\text {visual }} r_{\text {visual }}+C
$$

where $C$ is a constant, and $r_{\text {combined }}, r_{\text {vestibular }}$, and $r_{\text {visaul }}$ are mean firing rates across a trial $(0-$ $1500 \mathrm{~ms}$ from stimulus onset) for the three cue conditions, respectively. The weights for single cue conditions, $w_{\text {vestibular }}$ and $w_{\text {visual }}$, were determined by the least-squares method and plotted against each other to evaluate the heterogeneity of choice signals in the population for both LIP data and the model (Supplementary Figure 7d).

\section{Fisher Information Analysis}

591 To compute Fisher information $\left({ }^{32}\right.$ Seung and Sompolinsky, 1993), the full covariance matrix of 592 the population responses is needed, but this requires simultaneously recording from hundreds of neurons, which is not accessible to us yet. Instead, we calculated the shuffled Fisher information, which corresponds to the information in a population of neurons in which correlations have been removed (typically via shuffling across trials, hence the name). Shuffled Fisher information is given

$$
I_{\text {shuffled }}=\sum_{i=1}^{N} \frac{f_{i}^{\prime 2}}{\sigma_{i}^{2}}
$$

598 where $N$ is the number of neurons in the population; for the $i$ th neuron, $f_{i}^{\prime}$ denotes the derivative 599 of its local tuning curve, and $\sigma_{i}^{2}$ denotes the averaged variance of its responses around $0^{\circ}$ heading. 600 The tuning curve $f_{i}$ was constructed from both correct and wrong trials grouped by heading angles, 601 using spike counts in 250-ms sliding windows (advancing in 10-ms steps), and its derivative $f_{i}^{\prime}$ 602 was obtained from the slope of a linear fit of $f_{i}$ against headings. The variance $\sigma_{i}^{2}$ was computed 603 for each heading angle and then averaged. To estimate the standard errors of $I_{\text {shuffled }}$, we used a 
604 bootstrap procedure in which random samples of neurons were drawn from the population by 605 resampling with replacement (1000 iterations). To compare the experimental data with the model, 606 we repeated all the above steps on artificial LIP neurons in the model M2 and M3 (see below), with 607 the inter-neuronal noise correlation being ignored as well (Figure 6).

609 Two caveats are noteworthy when interpreting the Fisher information results. First, since the slope 610 of tuning curve $f^{\prime}$ is squared in the right-hand side of equation (1), the Fisher information will 611 always be non-negative regardless of the sign of $f^{\prime}$. As a result, even when the motion speed was

612 zero at the beginning of a trial, the population Fisher information already had a positive value 613 because of the noisy tuning curves during that period. Second, since we ignored inter-neuronal 614 noise correlations, $I_{\text {shuffled }}$ is most likely very different from the true Fisher information and thus

615 its value is arbitrary $\left({ }^{33}\right.$ Series, et al., 2004). Nonetheless, if we assume the noise correlation 616 structure of LIP population is similar across cue conditions, we can still rely on the qualitative 617 temporal evolution of $I_{\text {shuffled }}$ to appreciate how multisensory signals are accumulated across time 618 and cues in LIP.

\section{Network Simulation of ilPPC Framework}

\section{The responses of visual and vestibular neurons closely approximate ilPPC}

622 As mentioned previously, an important assumption of ilPPC is that the amplitude of the sensory 623 tuning curves be proportional to the nuisance parameters (in our case visual speed and vestibular 624 acceleration) ( ${ }^{9}$ Beck, et al., 2008). To check whether this is the case for the visual neurons, we 625 analyzed the spatio-temporal tuning curves of neurons in area MSTd (data from $\left({ }^{19} \mathbf{G u}\right.$, et al., 2006)).

626 We noticed that, for some neurons, the average tuning curves are not fully consistent with the ilPPC 627 assumption (Supplementary Figure 6a). Briefly, the mean firing rate of an MSTd neuron at time $628 t$ in response to a visual stimulus with heading $\theta$ can be well captured by 


$$
f(\theta, t)=v(t)\left(A \exp \left[K\left(\cos \left(\theta-\theta_{i}\right)-1\right)\right]-C\right)+B
$$

630 where $\theta_{i}$ denotes the preferred heading of the neuron $i$ and $v(t)$ is the velocity profile; $A, K, C$, 631 and $B$ correspond to the amplitude, the width, the null inhibition, and the baseline of its tuning 632 curve, respectively. The ilPPC framework requires the $v(t)$ term to be separable, namely, $f(\theta, t)=$ $633 h(\theta) g(v(t))$, where $h(\theta)$ is a pure spatial component and $g(v(t))$ is a multiplicative gain function 634 ( ${ }^{9}$ Beck, et al., 2008; ${ }^{10}$ Ma, et al., 2006). In equation (2), this requirement is equivalent to $C=0$ 635 and $B=0$, however, we found that some MSTd neurons often had non-zero baselines $(C>0$ and $636 B>0$ ). This will be harmful to the optimality of the ilPPC framework because, for example, when $637 v(t)=0$ (and thus the sensory reliability is zero), MSTd neurons still tend to generate background 638 spikes, which will bring nothing but noise into the simply summed population activity of 639 downstream areas in an ilPPC network.

641 To estimate the information loss due to this deviation, we simulated a population of MSTd neurons 642 with heterogeneous spatio-temporal tuning curves similar to what has been found experimentally 643 ( ${ }^{19} \mathbf{G u}$, et al., 2006). We calculated the information that can be decoded from the population by a series of optimal decoders $I_{\text {optimal }}$ and that can be recovered by the ilPPC solution $I_{\text {ilPPC }}$. We assumed that the population responses in MSTd contains differential correlations $\left({ }^{28}\right.$ Moreno-Bote, et al., 2014) such that the discrimination threshold of an ideal observer of MSTd activity was of the same order as the animal's performance. Under such conditions, we found that the information 648 loss $\left(I_{\text {optimal }}-I_{\text {ilPPC }}\right) / I_{\text {optimal }}$ was around $5 \%$. Detailed calculations of information loss are 649 provided in the Supplementary Materials. Therefore, the population response of MSTd neurons 650 provide a close approximation to an ilPPC, in the sense that simply summing the activity of MSTd neurons over time preserve $95 \%$ of the information conveyed by these neurons. 
654 (2) above still provides a good approximation to vestibular tuning curves, except that $C$ is close to 655 zero for most neurons $\left({ }^{37}\right.$ Laurens, et al., 2017), in which case the information less is even less 656 pronounced.

Network Model Implementing the ilPPC solution (Model M1)

659 We extended a previous ilPPC network model for unisensory decision making ( ${ }^{9}$ Beck, et al., 2008) 660 to our multisensory decision-making task. Two sensory layers, the vestibular layer and the visual layer, contained 100 linear-nonlinear-Poisson (LNP) neurons with bell-shape tuning curves to the of firing a spike at time step $\left[t_{n}-\delta t, t_{n}\right]$ was given by

$$
p\left(r_{i}^{\bullet}\left(t_{n}\right)=1\right)=\left[\delta t\left(g \bullet(t)\left(A \exp \left[K\left(\cos \left(\theta-\theta_{i}\right)-1\right)\right]-C\right)+B\right)+n_{i}\right]^{+}
$$
correlated noise term, and $[\cdot]^{+}$is the threshold-linear operator: $[x]^{+}=\max (x, 0)$. The spatial tuning was gain-modulated by a time-dependent function $g_{\bullet}(t)$, which modeled the reliability of the sensory evidence and took the form

$$
g_{\mathrm{VEST}}(t)=c_{\mathrm{VEST}}|\hat{a}(t)|, \quad g_{\mathrm{VIS}}(t)=c_{\mathrm{VIS}} \hat{v}(t)
$$

670 in which $\hat{a}(t)$ and $\hat{v}(t)$ are the same acceleration and velocity profiles as the experiments but with

671 the maximum values normalized to 1 , respectively, whereas $c_{\mathrm{VEST}}$ and $c_{\mathrm{VIS}}$ are scaling parameters 672 used to control the signal-to-noise ratio of sensory inputs and to balance the behavior performance 673 between the two cue conditions like in the experiments. The noise $n_{i}$ in equation (3) was generated 674 by convolving independent Gaussian noise with a circular Gaussian kernel,

$$
n_{i}=\sum_{j} A_{\eta} \exp \left(K_{\eta}\left(\cos \left(\theta_{i}-\theta_{j}\right)-1\right)\right) \eta_{j}
$$
we used were: $A=60 \mathrm{~Hz}, K=1.5, C=10 \mathrm{~Hz}, B=20 \mathrm{~Hz}, c_{\mathrm{VEST}}=c_{\mathrm{VIS}}=2.4, \delta t=1 \mathrm{~ms}$. 
678 Note that in equation (3), the gain $g_{\bullet}(t)$ cannot be factored out because $B>0$, which is the same

679 case as in MSTd (equation (2)). Accordingly, the neural code of M1's sensory layers is not exact

680 ilPPC ( ${ }^{9}$ Beck, et al., 2008). However, it is still a close approximation to ilPPC, since we have shown

681 in the previous section that MSTd is 95\% ilPPC-compatible.

The two sensory layers then projected to 100 LNP neurons in the integrator layer. We distinguished the integrator layer from the LIP layer because there are reasons to believe that LIP reflects the integration of the evidence but may not implement the integration per se $\left({ }^{27} \mathbf{K a t z}\right.$, et al., 2016). The integrator layer summed the sensory responses across both cues and time,

$$
m_{i}^{\mathrm{INT}}\left(t_{n+1}\right)=m_{i}^{\mathrm{INT}}\left(t_{n}\right)+g_{s t i m}\left(t_{n}\right)\left(\sum_{j} W_{i j}^{\mathrm{INTVEST}} r_{j}^{\mathrm{VEST}}\left(t_{n}\right)+\sum_{j} W_{i j}^{\mathrm{INTVIS}} r_{j}^{\mathrm{VIS}}\left(t_{n}\right)\right)
$$

where $m_{i}^{\mathrm{INT}}$ denotes the membrane potential proxy of neuron $i, W_{i j}^{\mathrm{INTVEST}}$ and $W_{i j}^{\mathrm{INTVIS}}$ are matrices for the feedforward weights from the vestibular and visual layer to the integrator layer, simulated one of the known circuit solutions to this problem $\left({ }^{\mathbf{5 2}} \mathbf{G o l d m a n , 2 0 0 9 )}\right.$, but this would not have affected our results, while making the simulation considerably more complicated. two saccade targets, i.e., neurons preferring $-90^{\circ}$ and $+90^{\circ}$ in the integrator layer, respectively, by

$$
W_{i j}^{\mathrm{INT} \bullet}=a \exp \left(k\left(\cos \left(\theta_{i}^{\mathrm{INT}}-\hat{\theta}\right)-1\right)\right)\left|\sin \left(\theta_{j}^{\bullet}\right)\right|
$$

698 in which a step function $\hat{\theta}$ controls the mapping,

$$
\hat{\theta}=\left\{\begin{array}{rl}
-\pi / 2, & \text { if } \theta_{j} \leq 0 \\
\pi / 2, & \text { if } \theta_{j}>0
\end{array} .\right.
$$


was used to determine the probability of the $i$ th integrator neuron firing a spike between times $t_{n}$ and $t_{n}+\delta t$,

$$
p\left(r_{i}^{\mathrm{INT}}\left(t_{n}\right)=1\right)=\left[m_{i}^{\mathrm{INT}}\left(t_{n}\right)\right]^{+} .
$$

Finally, the LIP layer received excitatory inputs from the integrator layer, together with visual inputs triggered by the two saccade targets (sent from the target layer). In addition, there were also

707 lateral connections in LIP to prevent saturation. In the linear step, the membrane potential proxy of 708 the $i$ th LIP neuron followed $m_{i}^{\mathrm{LIP}}\left(t_{n+1}\right)=\left(1-\frac{\delta t}{\tau}\right) m_{i}^{\mathrm{LIP}}\left(t_{n}\right)+\frac{1}{\tau}\left(\sum_{j} W_{i j}^{\mathrm{LIPINT}} r_{j}^{\mathrm{INT}}\left(t_{n}\right)+\sum_{j} W_{i j}^{\mathrm{LIPTARG}} r_{j}^{\mathrm{TARG}}\left(t_{n}\right)+\sum_{j} W_{i j}^{\mathrm{LIP}} r_{j}^{\mathrm{LIP}}\left(t_{n}\right)\right)(5)$ where the time constant, $\tau$, was set to $100 \mathrm{~ms} ; W_{i j}^{\mathrm{LIPINT}}$ and $W_{i j}^{\mathrm{LIPTAR}}$ are weight matrices for the

711 feedforward connections from the integrator layer and the target layer to the LIP layer, respectively, 712 and $W_{i j}^{\mathrm{LIP}}$ is the matrix for the recurrent connections within LIP. We used translation-invariant 713 weights for all these connections,

715 For $W_{i j}^{\mathrm{LIPINT}}$, we used $a=15, k=10, b=-3.6$; for $W_{i j}^{\mathrm{LIPTARG}}$, we used $a=8, k=5, b=0$;

716 and for $W_{i j}^{\mathrm{LIP}}$, we used $a=5, k=10, b=-3$. The term $r_{j}^{\mathrm{TARG}}\left(t_{n}\right)$ in equation (5) denotes the

717 visual response of the $j$ th neuron in the target layer induced by the two saccade targets,

$$
p\left(r_{j}^{\mathrm{TAR}}\left(t_{n}\right)=1\right)=s_{\text {targ }}\left(t_{n}\right) \sum_{m=1}^{2} p_{\text {targ }} \exp \left(k_{\text {targ }}\left(\cos \left(\theta_{j}^{\mathrm{TAR}}-\theta_{m}\right)-1\right)\right)
$$

where $\theta_{1}=-\pi / 2$ and $\theta_{2}=\pi / 2, p_{\text {targ }}=0.050$, and $k_{\text {targ }}=4$. The term $s_{\text {targ }}\left(t_{n}\right)$ modeled the saliency of the targets: $s_{\text {targ }}\left(t_{n}\right)=1$ before stimulus onset and $s_{\text {targ }}\left(t_{n}\right)=0.6$ afterwards.

722 After the linear step done in equation (5), the probability of observing a spike from the $i$ th LIP 


$$
p\left(r_{j}^{\mathrm{LIP}}\left(t_{n+1}\right)=1\right)=\left[m_{i}^{\mathrm{LIP}}\left(t_{n+1}\right)\right]^{+} .
$$

727 To let the model make decisions, we endowed it with a stopping bound such that the evidence 728 integration terminated when the peak activity in the LIP layer reached a threshold value. This 729 mechanism generates premature decisions in our fixed duration task, which have been observed in 730 the previous experiments $\left({ }^{29} \mathbf{K i a n i}\right.$, et al., 2008) as well as ours (see the main text). Specifically, 731 once the firing rate of any neuron in the LIP layer (determined from equation (6)) exceeded $\Theta^{\bullet}=$ $73237 \mathrm{~Hz}$ for a vestibular or a visual trial and $\Theta^{\mathrm{COMB}}=42 \mathrm{~Hz}$ for a combined trial, we blocked the 733 sensory inputs to the integrator layer by setting the gain factor in equation (4) to zero:

$$
g_{\text {stim }}\left(t_{n}\right)= \begin{cases}1, & \text { if } t_{n}<t_{\Theta} \\ 0, & \text { if } t_{n} \geq t_{\Theta}\end{cases}
$$

where $t_{\Theta}$ denotes the time of bound crossing. The instantaneous population activity at this time point $\boldsymbol{r}^{\mathrm{LIP}}\left(t_{\Theta}\right)$ was then used to determine the model's choice, while the network dynamics continued to evolve until the end of the 1.5 -s trial.

739 To read out the model's choice, we trained a linear support vector machine (SVM) to classify the

740 heading direction from $\boldsymbol{r}^{\mathrm{LIP}}\left(t_{\Theta}\right)$. We ran the network for 100 trials, used $\boldsymbol{r}^{\mathrm{LIP}}\left(t_{\Theta}\right)$ in 30 trials to 741 train the SVM, and then applied the SVM on the remaining 70 trials to make decisions and generate 742 psychometric functions of the model (with bootstrap 1000 times, Figure 5a and Supplementary 743 Figure 7a). The SVM acts like (or even outperforms) a local optimal linear estimator (LOLE) 744 trained by gradient descent $\left({ }^{33}\right.$ Series, et al., 2004). Importantly, such decoders could be 745 implemented with population codes in a biologically realistic point attractor network tuned for 746 optimal action selection in a discrimination task ( ${ }^{53}$ Deneve, et al., 1999), which could correspond 747 to downstream areas such as the motor layer of the superior colliculus ( ${ }^{9}$ Beck, et al., 2008). 
750 In model M2, we generalized the homogeneous ilPPC network described above (model M1) to a

751 heterogeneous one. Instead of taking perfect sums like in model M1, neurons in the integration

752 layer of the model computed random linear combinations of vestibular and visual inputs. It is

753 indeed been widely shown that integration weights in vivo are heterogeneous and are well-captured

754 by "long-tailed" lognormal distributions (see for example $\left({ }^{54}\right.$ Song, et al., 2005)). To simulate this

755 in $\mathrm{M} 2$, we drew each synaptic weight $w_{\mathrm{M} 2}$ in $\mathrm{M} 2$ from a lognormal distribution

$$
p\left(w_{\mathrm{M} 2}=x\right)=\frac{1}{\sqrt{2 \pi} \sigma x} \exp \left(-\frac{(\log x-\mu)^{2}}{2 \sigma^{2}}\right)
$$

where $\mu$ and $\sigma$ were chosen such that the expectation $e\left(w_{\mathrm{M} 2}\right)$ and the standard deviation $s\left(w_{\mathrm{M} 2}\right)$

of $w_{\mathrm{M} 2}$ were both equal to its counterpart synaptic weight $w_{\mathrm{M} 1}$ in model M1:

$$
e\left(w_{\mathrm{M} 2}\right)=s\left(w_{\mathrm{M} 2}\right)=w_{\mathrm{M} 1} \text {. }
$$

The parameters $\mu$ and $\sigma$ in equation (7) were related to $e$ and $s$ through

$$
\begin{aligned}
& \mu=\log \left(e^{2} / \sqrt{e^{2}+s^{2}}\right) \\
& \sigma=\sqrt{\log \left(s^{2} / e^{2}+1\right)} .
\end{aligned}
$$

762 If $w_{\mathrm{M} 1}<0$, a negative sign was added to the resulting $w_{\mathrm{M} 2}$, since lognormal distributions are 763 always non-negative.

\section{Network with Short Integration Time Constant (M3)}

766 We also simulated a sub-optimal model M3 in which the network does not integrate evidence over

767 time. This was done by replacing equation (4) with

$768 \quad m_{i}^{\mathrm{INT}}\left(t_{n+1}\right)=\left(1-\frac{\delta t}{\tau}\right) m_{i}^{\mathrm{INT}}\left(t_{n}\right)+\frac{1}{\tau} g_{s t i m}\left(t_{n}\right)\left(\sum_{j} W_{i j}^{\mathrm{INTVEST}} r_{j}^{\mathrm{VEST}}\left(t_{n}\right)+\sum_{j} W_{i j}^{\mathrm{INTVIS}} r_{j}^{\mathrm{VIS}}\left(t_{n}\right)\right)$

769 where $\tau=100 \mathrm{~ms}$ and other terms are the same as in equation (4). 
772 To test whether the responses of the optimal and homogeneous model M1 can be linearly 773 reproduced from responses of M2, M3, and the experimental data, we first calculated the "optimal 774 traces" from M1 (Figure 5b), using

$$
\Delta P S T H_{\mathrm{M} 1}^{\bullet}=<P S T H_{M 1, i}^{\bullet,+}>-<P S T H_{M 1, i}^{\bullet,-}>
$$

Where - denotes three cue conditions (vestibular, visual, and combined), $P S T H_{M 1, i}^{\bullet,+}$ and $P S T H_{M 1, i}^{\bullet,-}$ denote averaged PSTH for the $i$ th LIP unit in the network M1 when the network makes

778 correct choices towards the neuron's preferred direction and null direction, respectively, and $<\cdot>$ 779 denotes averaging across cells. To mimic the experimental procedure, only cells whose preferred 780 directions were close to $\pm 90^{\circ}$ (with deviations less than $20^{\circ}$ ) were used. Similarly, we extracted 781 single cell activities from M2, M3, the LIP data, and the MSTd data $\left({ }^{19} \mathbf{G u}\right.$, et al., 2006)

$$
\triangle P S T H_{*, i}^{\bullet}=P S T H_{*, i}^{\bullet,+}-P S T H_{*, i}^{\bullet,-}
$$

783 where $* \in\{\mathrm{M} 2, \mathrm{M} 3, \mathrm{LIP}$ data, MSTd data $\}$. Then we optimized sets of linear weights $\boldsymbol{w}_{*}$ to 784 minimize the cost function

$$
E_{*}=\sum_{\bullet} \sum_{n}\left(\Delta P S T H_{\mathrm{M} 1}^{\bullet}\left(t_{n}\right)-\sum_{i} w_{*, i} \Delta P S T H_{*, i}^{\bullet}\left(t_{n}\right)\right)^{2}
$$

where, for example, $w_{\mathrm{LIP}, i}$ represents the weight of the neuron $i$ in the LIP data when a

787 downstream area reads out LIP dynamics linearly to reproduce the optimal traces. To reduce overfitting, we partitioned the data into two subsets along time by randomly assigning the time bins into two sets, one for fitting $\left(T_{\text {fit }}\right)$ and the other for validating $\left(T_{\text {valid }}\right)$. During fitting, when the

790 validating error $E_{*, t_{n} \in T_{\text {valid }}}$ started increasing, we stopped the iteration, a procedure known as early 791 stopping. The fitting results are shown in Figure 5c-f. Note that the $\triangle P S T H \mathrm{~s}$ in the cost function 792 (equation (8)) grouped all the heading angles together. The results were qualitatively similar when 793 the cost function included error terms calculated from each heading angle separately, i.e., 


$$
E_{*}=\sum_{\bullet} \sum_{n} \sum_{|h|}\left(\Delta P S T H_{\mathrm{M} 1}^{\bullet,|h|}\left(t_{n}\right)-\sum_{i} w_{*, i} \Delta P S T H_{*, i}^{\bullet,|h|}\left(t_{n}\right)\right)^{2}
$$

where $|h|$ denotes the absolute value of heading angle $\left(0^{\circ}, 1^{\circ}, 2^{\circ}, 4^{\circ}, 8^{\circ}\right)$. The reconstructions of

M1 traces with LIP activities using equation (9) are shown in Supplementary Figure 9.

798 To assess the robustness of the linear reconstruction, we randomly subsampled the same number of 799 neurons $(\mathrm{n}=50$, without replacement) from the four data sets, performed the linear fitting, and repeated this procedure for 1000 times. The mean squared error and the distribution of readout weights of the fittings are shown in Figure 5g, h. To examine whether only a small fraction of cells contributed heavily to the fittings or whether the majority of cells did, we compared the distributions of weights from the four data sets with the distribution of weights from a random linear decoder. To do so, for each subsampling, we also generated a set of random readout weights from a rectified Gaussian distribution (Figure 5h, black curve) and computed the kurtosis of the distribution of weights from the random decoder as well as those from the four data sets (Figure 5i). The p-values were derived from the empirical subsampling distributions (two-tailed).

\section{Data and Code Availability}

810 MATLAB code for the network model and the information loss calculation is available at the 811 following public repository: https://github.com/hanhou/Multisensory-PPC. Experimental data and 812 code for data analysis are available upon request to the authors.

\section{Acknowledgments}

815 We thank Jianyu Lu, Zhao Zeng, and Xuefei Yu for collecting part of the MSTd data, Wenyao Chen 816 for monkey care and training, and Ying Liu for $\mathrm{C}++$ software programming. This work was 817 supported by grants from the National Natural Science Foundation of China Project (31761133014), 
818 the Strategic Priority Research Program of CAS (XDBS01070201), the Shanghai Municipal

819 Science and Technology Major Project (2018SHZDZX05) to Y.G and by grants from the Simons

820 Collaboration for the Global Brain and the Swiss National Science Foundation (\#31003A_165831)

821 to A.P.

822

823 Author Contributions

824 H.H. and Y.G. conceived the project and designed the experiments. H.H., Q.Z., and Y.Z. performed

825 the experiments. H.H. analyzed the data. H.H. and A.P. developed the models and implemented the

826 simulations. H.H., A.P., and Y.G. wrote the manuscript.

828 Competing financial interests

829 The authors declare no competing financial interests.

831 References

832 1. Ratcliff, R. A theory of memory retrieval. Psychological review 85, 59 (1978).

833 2. Ratcliff, R. \& McKoon, G. The diffusion decision model: theory and data for two-choice decision 834 tasks. Neural computation 20, 873-922 (2008).

835 3. Ratcliff, R. \& Rouder, J.N. Modeling response times for two-choice decisions. Psychological 836 science 9, 347-356 (1998).

837 4. Ratcliff, R. \& Smith, P.L. A comparison of sequential sampling models for two-choice reaction 838 time. Psychol Rev 111, 333-367 (2004).

839 5. Laming, D.R.J. Information theory of choice-reaction times. (1968).

840 6. Bogacz, R., Brown, E., Moehlis, J., Holmes, P. \& Cohen, J.D. The physics of optimal decision 841 making: a formal analysis of models of performance in two-alternative forced-choice tasks. Psychol 842 Rev 113, 700-765 (2006).

843 7. Gold, J.I. \& Shadlen, M.N. The neural basis of decision making. Annual review of neuroscience 844 30, 535-574 (2007).

845 8. Drugowitsch, J., DeAngelis, G.C., Klier, E.M., Angelaki, D.E. \& Pouget, A. Optimal 846 multisensory decision-making in a reaction-time task. Elife 3 (2014).

847 9. Beck, J.M., et al. Probabilistic population codes for Bayesian decision making. Neuron 60, 1142$848 \quad 1152(2008)$.

849 10. Ma, W.J., Beck, J.M., Latham, P.E. \& Pouget, A. Bayesian inference with probabilistic 850 population codes. Nat Neurosci 9, 1432-1438 (2006).

851 11. Fetsch, C.R., Pouget, A., DeAngelis, G.C. \& Angelaki, D.E. Neural correlates of reliability852 based cue weighting during multisensory integration. Nat Neurosci 15, 146-154 (2012). 
12. Gu, Y., Angelaki, D.E. \& Deangelis, G.C. Neural correlates of multisensory cue integration in macaque MSTd. Nat Neurosci 11, 1201-1210 (2008).

13. Fetsch, C.R., DeAngelis, G.C. \& Angelaki, D.E. Bridging the gap between theories of sensory cue integration and the physiology of multisensory neurons. Nature reviews. Neuroscience 14, 429442 (2013).

14. Shadlen, M.N. \& Newsome, W.T. Neural basis of a perceptual decision in the parietal cortex (area LIP) of the rhesus monkey. Journal of neurophysiology 86, 1916-1936 (2001).

15. Shadlen, M.N. \& Newsome, W.T. Motion perception: seeing and deciding. Proceedings of the National Academy of Sciences of the United States of America 93, 628-633 (1996).

16. Huk, A.C., Katz, L.N. \& Yates, J.L. The Role of the Lateral Intraparietal Area in (the Study of) Decision Making. Annual review of neuroscience 40, 349-372 (2017).

17. Roitman, J.D. \& Shadlen, M.N. Response of neurons in the lateral intraparietal area during a combined visual discrimination reaction time task. The Journal of neuroscience : the official journal of the Society for Neuroscience 22, 9475-9489 (2002).

18. Boussaoud, D., Ungerleider, L.G. \& Desimone, R. Pathways for Motion Analysis - Cortical Connections of the Medial Superior Temporal and Fundus of the Superior Temporal Visual Areas in the Macaque. J Comp Neurol 296, 462-495 (1990).

19. Gu, Y., Watkins, P.V., Angelaki, D.E. \& DeAngelis, G.C. Visual and nonvisual contributions to three-dimensional heading selectivity in the medial superior temporal area. The Journal of neuroscience : the official journal of the Society for Neuroscience 26, 73-85 (2006).

20. Chen, A., DeAngelis, G.C. \& Angelaki, D.E. Representation of vestibular and visual cues to self-motion in ventral intraparietal cortex. The Journal of neuroscience : the official journal of the Society for Neuroscience 31, 12036-12052 (2011c).

21. Chen, A., DeAngelis, G.C. \& Angelaki, D.E. Functional Specializations of the Ventral Intraparietal Area for Multisensory Heading Discrimination. The Journal of Neuroscience 33, 3567-3581 (2013).

22. Nikbakht, N., Tafreshiha, A., Zoccolan, D. \& Diamond, M.E. Supralinear and Supramodal Integration of Visual and Tactile Signals in Rats: Psychophysics and Neuronal Mechanisms. Neuron 97, 626-639.e628 (2018).

23. Raposo, D., Kaufman, M.T. \& Churchland, A.K. A category-free neural population supports evolving demands during decision-making. Nat Neurosci 17, 1784-1792 (2014).

24. Knill, D.C. \& Richards, W. Perception as Bayesian inference (Cambridge University Press, 1996).

25. Park, I.M., Meister, M.L., Huk, A.C. \& Pillow, J.W. Encoding and decoding in parietal cortex during sensorimotor decision-making. Nat Neurosci 17, 1395-1403 (2014).

26. Meister, M.L., Hennig, J.A. \& Huk, A.C. Signal multiplexing and single-neuron computations in lateral intraparietal area during decision-making. The Journal of Neuroscience 33, 2254-2267 (2013).

27. Katz, L.N., Yates, J.L., Pillow, J.W. \& Huk, A.C. Dissociated functional significance of decision-related activity in the primate dorsal stream. Nature advance online publication (2016). 28. Moreno-Bote, R., et al. Information-limiting correlations. Nat Neurosci 17, 1410-1417 (2014). 29. Kiani, R., Hanks, T.D. \& Shadlen, M.N. Bounded integration in parietal cortex underlies decisions even when viewing duration is dictated by the environment. The Journal of neuroscience : the official journal of the Society for Neuroscience 28, 3017-3029 (2008).

30. Scott, B.B., et al. Fronto-parietal Cortical Circuits Encode Accumulated Evidence with a Diversity of Timescales. Neuron 95, 385-398.e385 (2017).

31. Beck, J., Bejjanki, V.R. \& Pouget, A. Insights from a simple expression for linear fisher information in a recurrently connected population of spiking neurons. Neural computation 23, 1484-1502 (2011).

32. Seung, H.S. \& Sompolinsky, H. Simple models for reading neuronal population codes. Proceedings of the National Academy of Sciences of the United States of America 90, 10749-10753 
904

905

906

907

908

909

910

911

912

913

914

915

916

917

918

919

920

921

922

923

924

925

926

927

928

929

930

931

932

933

934

935

936

937

938

939

940

941

942

943

944

945

946

947

948

949

950

951

952

953

954

(1993).

33. Series, P., Latham, P.E. \& Pouget, A. Tuning curve sharpening for orientation selectivity: coding efficiency and the impact of correlations. Nat Neurosci 7, 1129-1135 (2004).

34. Chen, A., DeAngelis, G.C. \& Angelaki, D.E. A comparison of vestibular spatiotemporal tuning in macaque parietoinsular vestibular cortex, ventral intraparietal area, and medial superior temporal area. The Journal of neuroscience : the official journal of the Society for Neuroscience 31, 30823094 (2011a).

35. Fetsch, C.R., et al. Spatiotemporal Properties of Vestibular Responses in Area MSTd. Journal of neurophysiology 104, 1506-1522 (2010).

36. Smith, A.T., Greenlee, M.W., DeAngelis, G.C. \& Angelaki, D.E. Distributed Visual-Vestibular Processing in the Cerebral Cortex of Man and Macaque. Multisensory Research 30, 91-120 (2017). 37. Laurens, J., et al. Transformation of spatiotemporal dynamics in the macaque vestibular system from otolith afferents to cortex. Elife 6, e20787 (2017).

38. Lisberger, S.G. \& Movshon, J.A. Visual motion analysis for pursuit eye movements in area MT of macaque monkeys. The Journal of neuroscience : the official journal of the Society for Neuroscience 19, 2224-2246 (1999).

39. Churchland, A.K., et al. Variance as a signature of neural computations during decision making. Neuron 69, 818-831 (2011).

40. Bizley, J.K., Jones, G.P. \& Town, S.M. Where are multisensory signals combined for perceptual decision-making? Current opinion in neurobiology 40, 31-37 (2016).

41. Gu, Y. Vestibular signals in primate cortex for self-motion perception. Current opinion in neurobiology 52, 10-17 (2018).

42. Deneve, S., Latham, P.E. \& Pouget, A. Efficient computation and cue integration with noisy population codes. Nat Neurosci 4, 826-831 (2001).

43. Gu, Y., Deangelis, G.C. \& Angelaki, D.E. Causal links between dorsal medial superior temporal area neurons and multisensory heading perception. The Journal of neuroscience : the official journal of the Society for Neuroscience 32, 2299-2313 (2012).

44. Chen, A., Gu, Y., Liu, S., DeAngelis, G.C. \& Angelaki, D.E. Evidence for a Causal Contribution of Macaque Vestibular, But Not Intraparietal, Cortex to Heading Perception. The Journal of neuroscience : the official journal of the Society for Neuroscience 36, 3789-3798 (2016).

45. Chandrasekaran, C. Computational principles and models of multisensory integration. Current opinion in neurobiology 43, 25-34 (2017).

46. Wong, K.F. \& Wang, X.J. A recurrent network mechanism of time integration in perceptual decisions. The Journal of neuroscience : the official journal of the Society for Neuroscience 26, 1314-1328 (2006).

47. Wang, X.J. Probabilistic decision making by slow reverberation in cortical circuits. Neuron 36, 955-968 (2002).

48. Mante, V., Sussillo, D., Shenoy, K. \& Newsome, W. Context-dependent computation by recurrent dynamics in prefrontal cortex. Nature 503, 78-84 (2013).

49. Song, H.F., Yang, G.R. \& Wang, X.J. Reward-based training of recurrent neural networks for cognitive and value-based tasks. Elife 6 (2017).

50. Barash, S., Bracewell, R.M., Fogassi, L., Gnadt, J.W. \& Andersen, R.A. Saccade-related activity in the lateral intraparietal area. I. Temporal properties; comparison with area 7a. Journal of neurophysiology 66, 1095-1108 (1991).

51. Gu, Y., Fetsch, C.R., Adeyemo, B., Deangelis, G.C. \& Angelaki, D.E. Decoding of MSTd population activity accounts for variations in the precision of heading perception. Neuron 66, 596609 (2010).

52. Goldman, M.S. Memory without Feedback in a Neural Network. Neuron 61, 621-634 (2009).

53. Deneve, S., Latham, P.E. \& Pouget, A. Reading population codes: a neural implementation of ideal observers. Nat Neurosci 2, 740-745 (1999).

54. Song, S., Sjostrom, P.J., Reigl, M., Nelson, S. \& Chklovskii, D.B. Highly nonrandom features 
bioRxiv preprint doi: https://doi.org/10.1101/480178; this version posted November 29, 2018. The copyright holder for this preprint (which was not certified by peer review) is the author/funder. All rights reserved. No reuse allowed without permission.

955 of synaptic connectivity in local cortical circuits. PLoS biology 3, e68 (2005).

956

957 


\section{Supplementary Materials}

959 Supplementary Figure 1. Recording sites and reliable area mapping

960 Supplementary Figure 2. More example LIP cells

961 Supplementary Figure 3. Task-difficulty dependence of choice signals

962 Supplementary Figure 4. Macaque LIP is category-free

963 Supplementary Figure 5. De-mixing of choice and modality signals

964 Supplementary Figure 6. Information loss of ilPPC solution with heterogeneous MSTd

965 population

966 Supplementary Figure 7. Model M2 achieves optimal behavior with heterogeneous units

967 Supplementary Figure 8. Example units in linear reconstruction of M1

968 Supplementary Figure 9. Linear reconstruction of M1 with cost function calculated for

969 separated heading angles

970 Supplementary Figure 10. A trained recurrent neural network (RNN) performing

971 multisensory decision-making task 
a
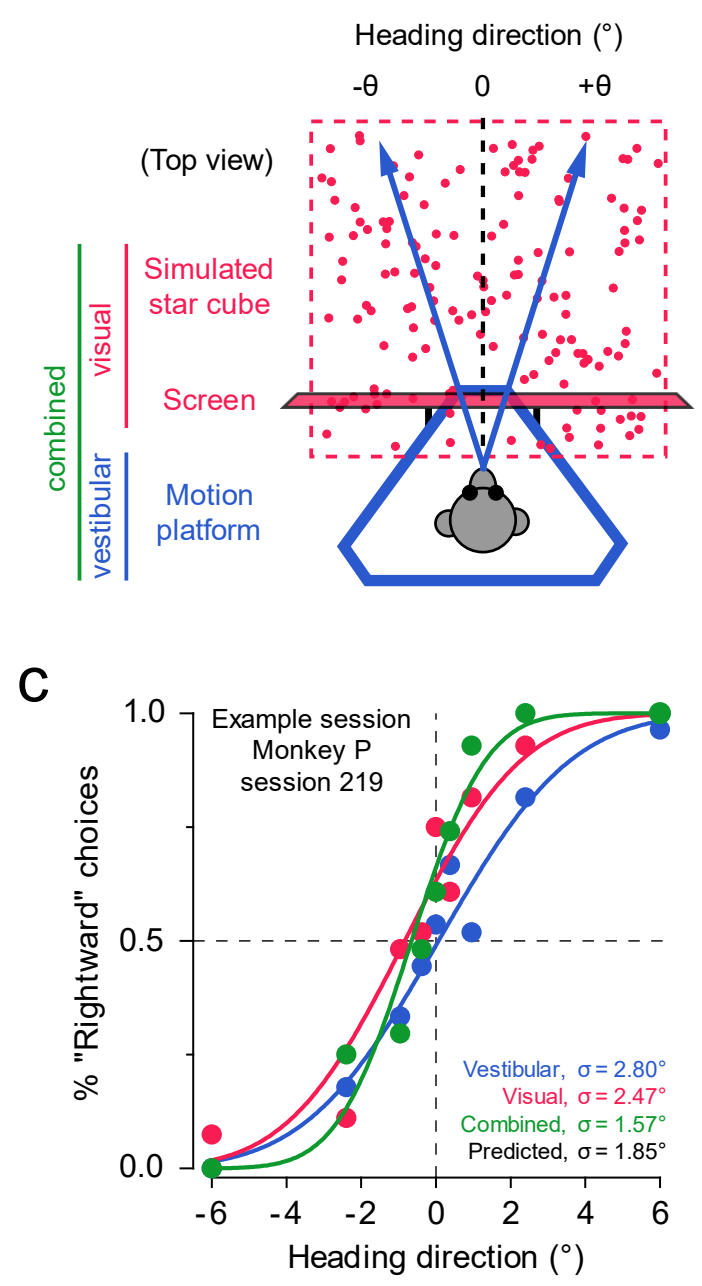

b

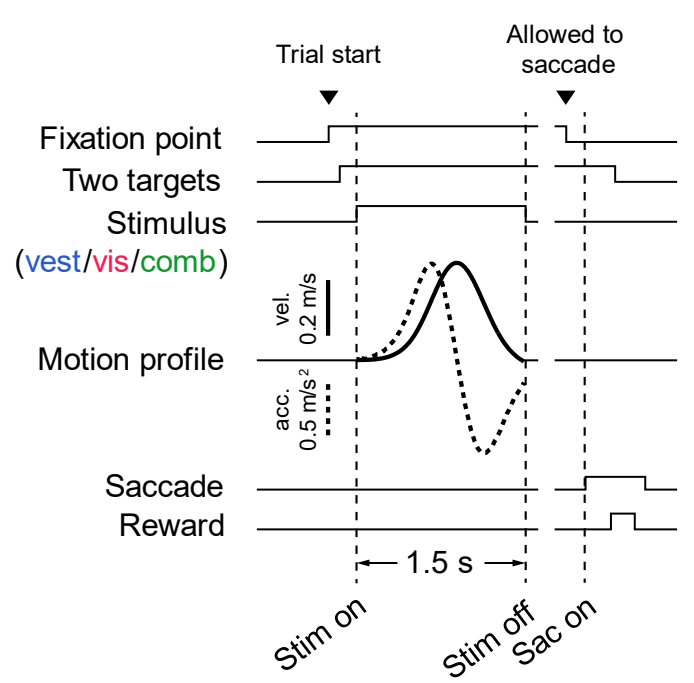

d

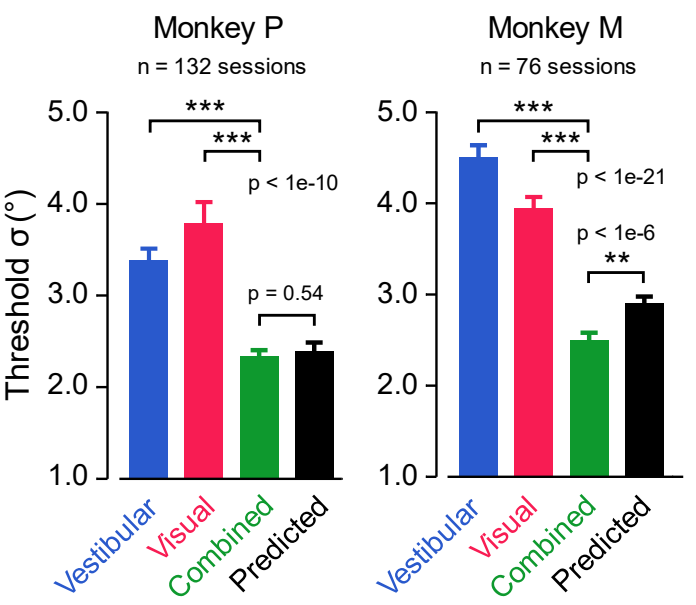

Figure 1 Optimal cue integration in vestibular-visual multisensory decision-making task.

(a) Schematic drawing of the experimental setup (top view). The vestibular (blue) and visual (red) stimuli of self-motion were provided by a motion platform and an LCD screen mounted on it, respectively. The monkey was seated on the platform and physically translated within the horizontal plane (blue arrows), whereas the screen rendered optical flow simulating what the monkey would see when moving through a three-dimensional star field (red dots). In a combined condition (green), both vestibular and visual stimuli were presented synchronously. The monkey's task was to discriminate whether the heading direction was to the left or the right of the straight ahead (black dashed line). (b) Task timeline. The monkey initiated a trial by fixating at a fixation point, and two choice targets appeared. The monkey then experienced a 1.5-s forward self-motion stimulus with a small leftward or rightward component, after which the monkey reported his perceived heading by making a saccadic eye movement to one of the two targets. The self-motion speed followed a Gaussian-shape profile. (c) Example psychometric functions from one session. The proportion of "rightward" choices is plotted against the headings for three cue conditions respectively. Smooth curves represent best-fitting cumulative Gaussian functions. (d) Average psychophysical thresholds from two monkeys for three conditions and predicted thresholds calculated from optimal cue integration theory (black bars). Error bars indicate s.e.m.; $p$ values were from paired t-test. 


\section{a}

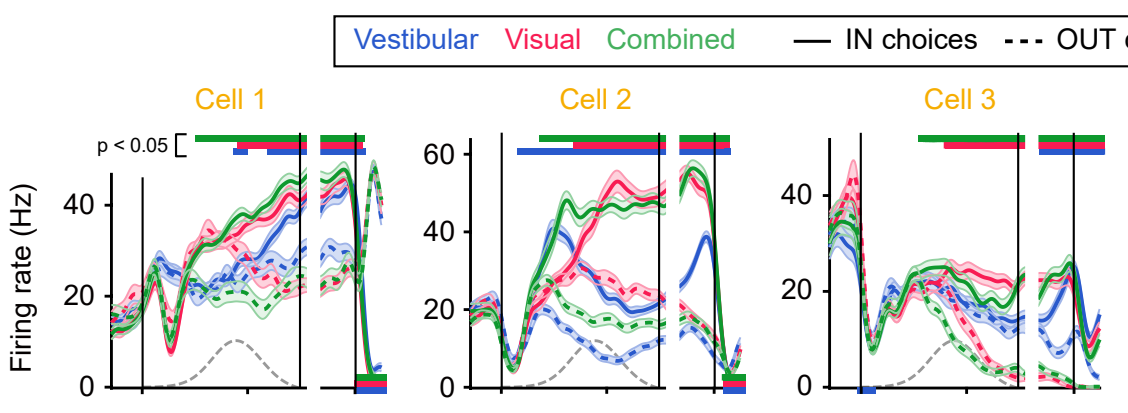

b
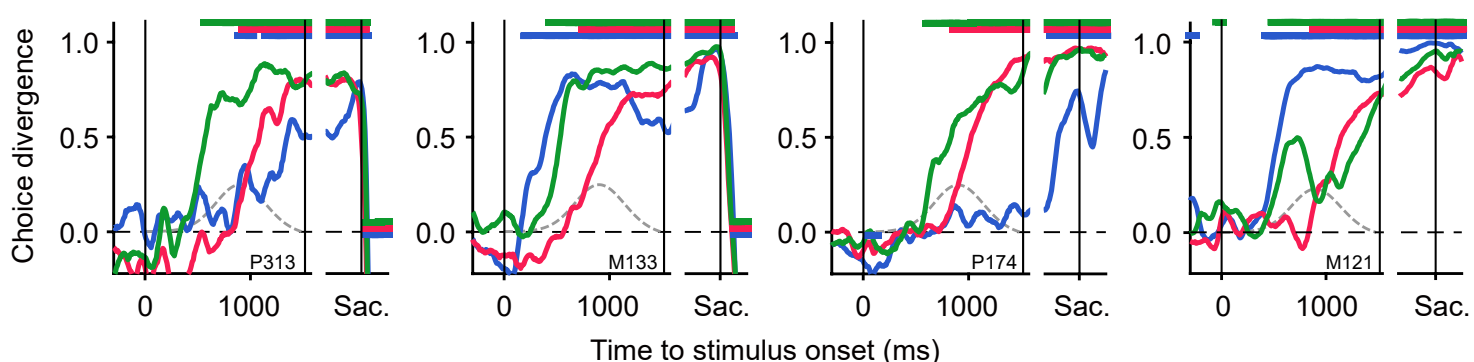

C

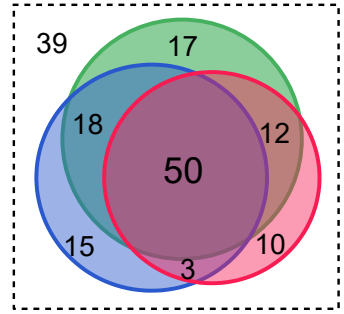

$\begin{array}{ll}\text { Total } & 164 \\ \text { Vestibular choice } & 86(52 \%) \\ \text { Visual choice } & 75(46 \%) \\ \text { Combined choice } & 97(59 \%) \\ \text { Any choice } & 125(76 \%) \\ \text { All choices } & 50(30 \%) \\ \text { None choice } & 39(24 \%)\end{array}$

Figure 2 Heterogeneous choice signals in LIP population.

(a) Peri-stimulus time histograms (PSTHs) of four examples cells. Spike trains were aligned to stimulus onset (left subpanels) and saccade onset (right subpanels), respectively, and grouped by cue condition and monkey's choice. Vestibular, blue; visual, red; combined, green. Toward the cell's response field (RF), or IN choices, solid curves; away from the cell's RF, or OUT choices, dashed curves. Mean firing rates were computed from 10-ms time windows and smoothed with a Gaussian $(\sigma=50 \mathrm{~ms})$; only correct trials or $0^{\circ}$ heading trials were included. Shaded error bands, s.e.m. Horizontal color bars represent time epochs in which IN and OUT trials have significantly different firing rates $(p<0.05$, t-test), with the color indicating cue condition and the position indicating the relationship between IN and OUT firings (IN > OUT, top; IN < OUT, bottom). Gray dashed curves represent the actual speed profile measured by an accelerometer attached to the motion platform. (b) Choice divergence (CD) of the same four cells. CD ranged from -1 to 1 and was derived from ROC analysis for PSTHs in each 10-ms window (see Methods). Horizontal color bars are the same as in a except that $p$-values were from permutation test $(n=1000)$. (c) Venn diagram showing the distribution of choice signals. Numbers within colored areas indicate the numbers of neurons that have significant grand CDs (CD computed from all spikes in 0-1500 ms) under the corresponding combinations of cue conditions. 


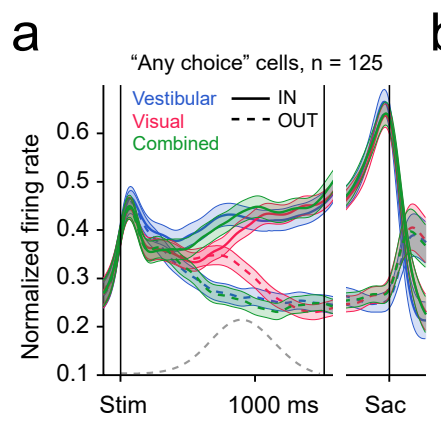

b

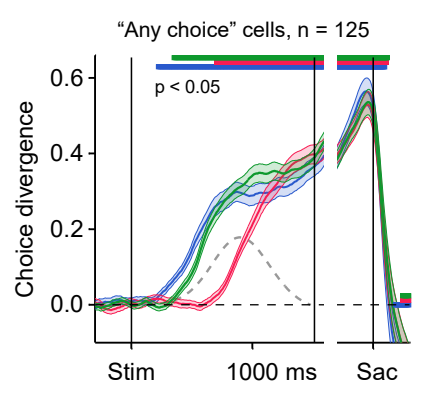

d

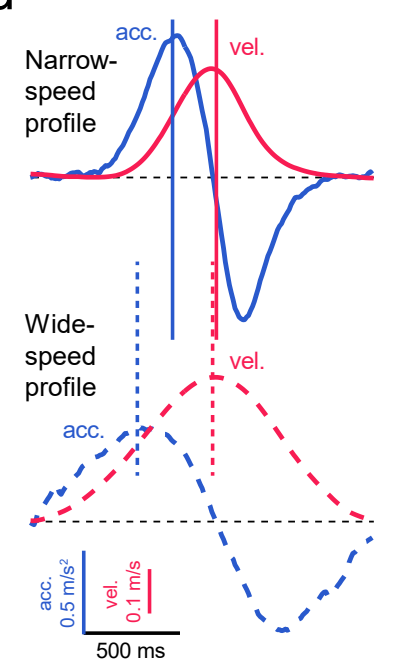

e

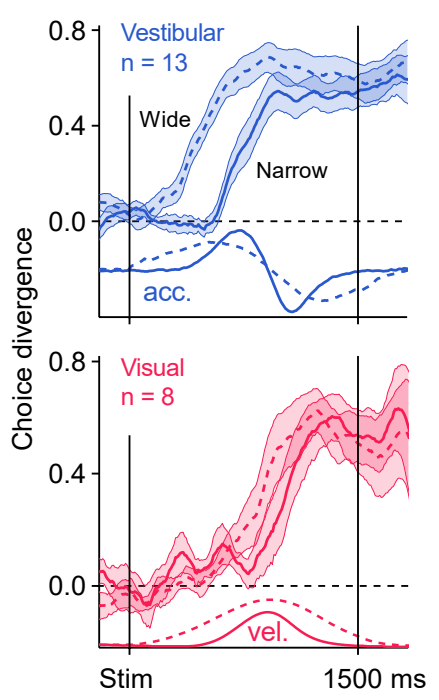

C

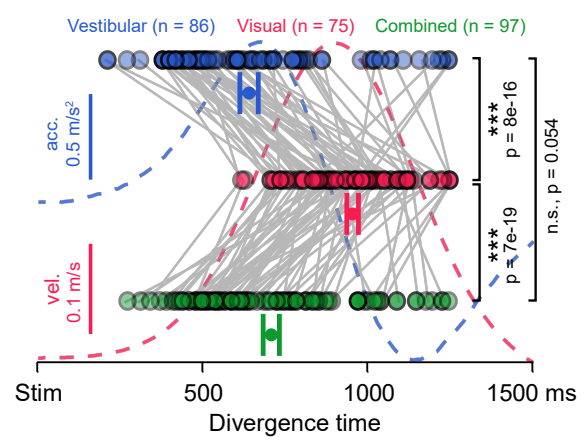

$f$

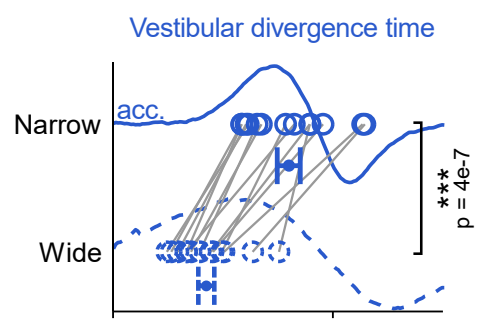

Visual divergence time

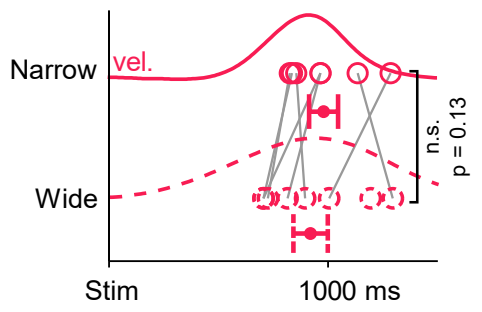

Figure 3 LIP integrates vestibular acceleration but visual speed.

(a and b) Population average of normalized PSTHs (a) and CD (b) from 125 "any choice" cells. The vestibular (blue) and combined (green) CDs ramped up much earlier than the visual one (red). Horizontal color bars indicate the time epochs in which population CDs are significantly larger than zero ( $p<0.05$, t-test). Gray dashed curve, the actual Gaussian speed profile; shaded error bands, s.e.m. (c) Divergence time of cells with significant grand CD for each condition. Divergence time was defined as the first occurrence of a $250-\mathrm{ms}$ window in which $C D$ was consistently larger than zero ( $p$ $<0.05$, permutation test). Gray lines connect data from the same cells; acceleration and speed profiles shown in the background. Data points with horizontal error bars, mean \pm s.e.m. of population divergence time; $p$ values, t-test. (d) Two motion profiles used to isolate contributions of acceleration and speed to LIP ramping. Top and solid, the narrow-speed profile; bottom and dashed, the widespeed profile; blue, acceleration; red, speed. Note that by widening the speed profile, we shifted the time of acceleration peak forward (blue vertical lines) while keeping the speed peak unchanged (red vertical lines). (e) Vestibular and visual CDs under the two motion profiles. (f) Comparison of divergence time between narrow and wide profiles. Note that the vestibular divergence time was significantly shifted, whereas the visual one was not, indicating that LIP integrates sensory evidence from vestibular acceleration and visual speed. 

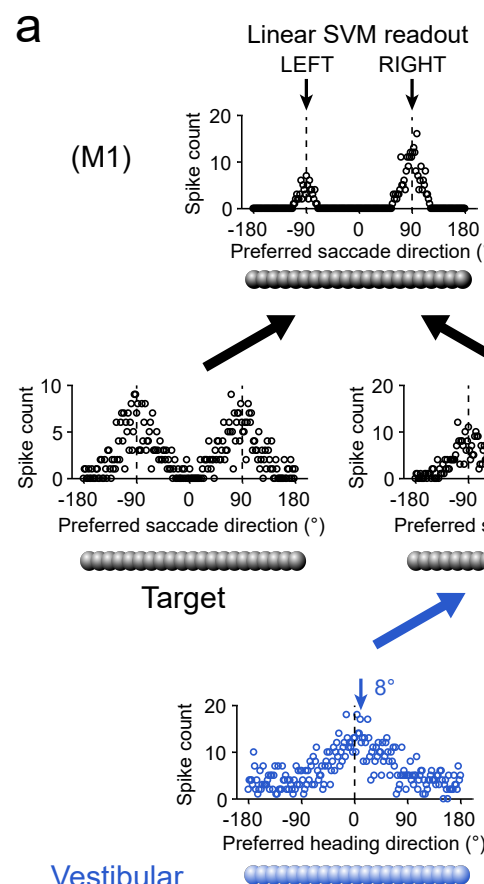

$M$ readout RIGHT RIGHT<smiles>[AlH][V]</smiles>

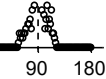
ade direction $\left(0^{\circ}\right)$ LIP

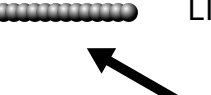

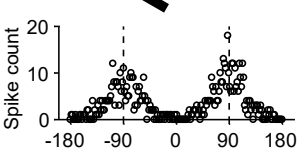
Preferred saccade direction $\left(^{\circ}\right)$ Integrator

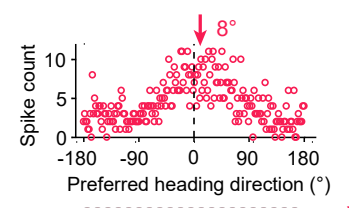

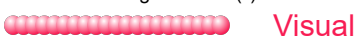

b

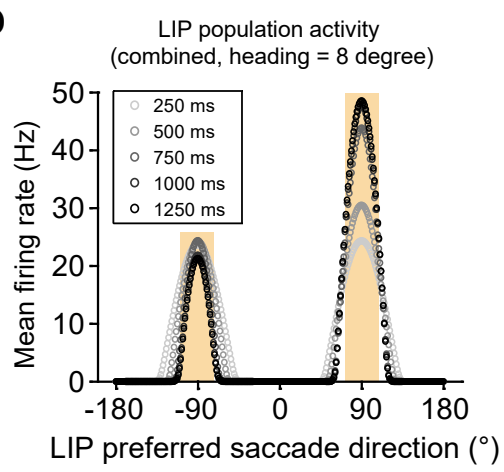

C

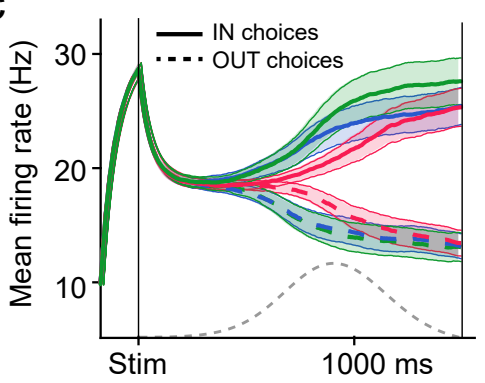

Figure 4 Neural network model with invariant linear probabilistic population codes (ilPPC).

(a) Network architecture of model M1. The model consists of three interconnected layers of linearnonlinear-Poisson units (inset). Units in Vestibular and Visual layers have bell-shape ilPPCcompatible tuning curves for heading direction and receive heading stimuli with temporal dynamics following acceleration and speed, respectively. The intermediate Integrator layer simply sums the incoming spikes from the two sensory layers over time and transforms the tuning curves for heading direction to that for saccade direction $\left(-90^{\circ}\right.$, leftward choice; $+90^{\circ}$, rightward choice). The LIP layer receives the integrated heading inputs from the Integrator layer, together with visual responses triggered by the two saccade targets. LIP units also have lateral connections implementing shortrange excitation and long-range inhibition. Once a decision boundary is hit, or when the end of the trial is reached (1.5 s), LIP activity is decoded by a linear support vector machine for action selection (see Methods). Circles indicate representative patterns of activity for each layer; spike counts from 800-1000 ms; combined condition, $8^{\circ}$ heading. (b) Population firing rate in the LIP layer at five different time points (the same stimulus as in a, averaged over 100 repetitions). (c) Average PSTHs across LIP population. Trials included three cue conditions and nine heading directions $\left( \pm 8^{\circ}, \pm 4^{\circ}, \pm 2^{\circ}\right.$, $\left.\pm 1^{\circ}, 0^{\circ}\right)$. To mimic the experimental procedure, only units with preferred saccade direction close to $\pm 90^{\circ}$ were used (with deviation less than $20^{\circ}$; yellow shaded area in $\mathbf{b}$ ). Notations are the same as in Figure $2 \mathrm{a}$ and Figure $\mathbf{3 a}$. 

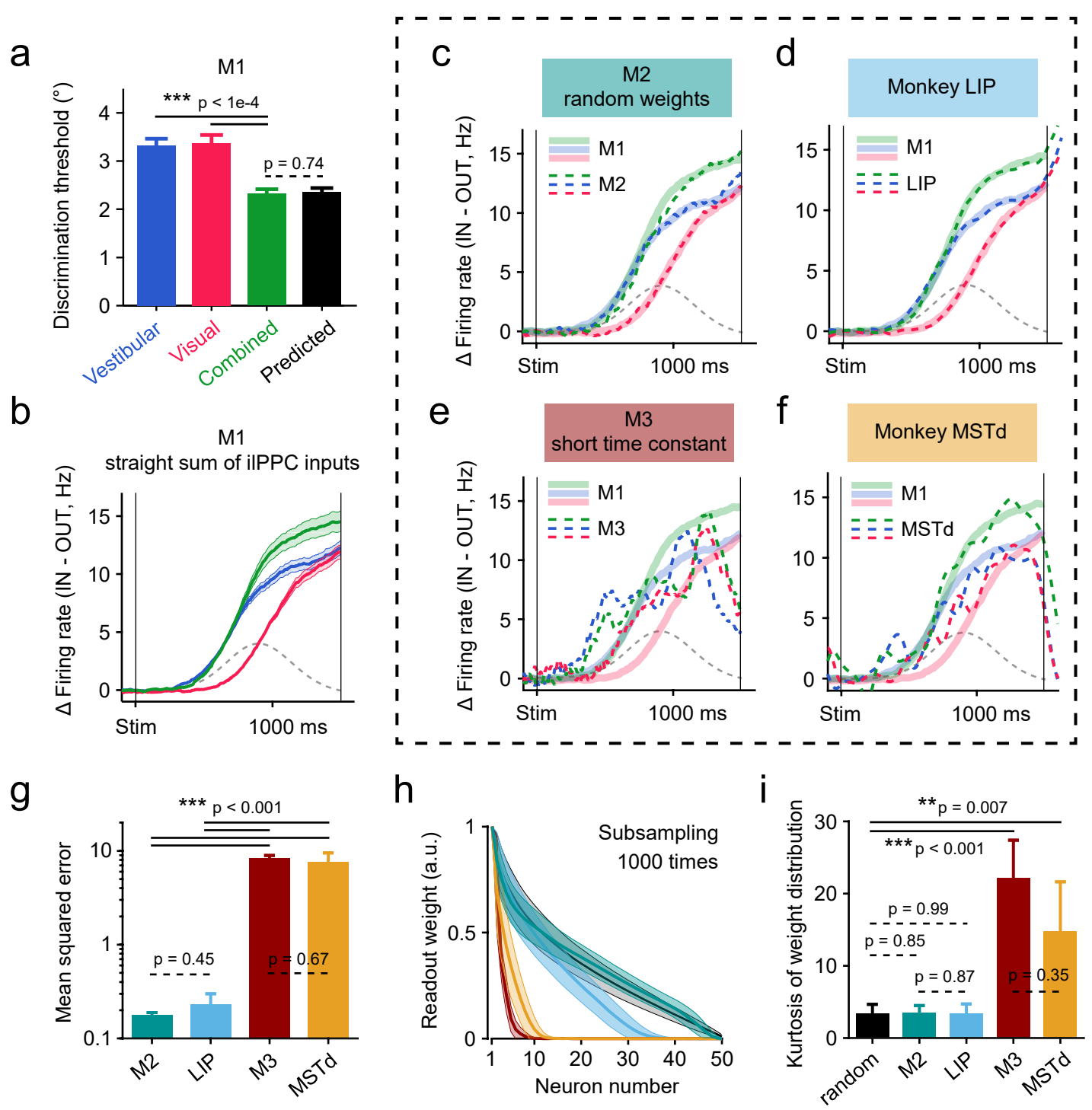

Figure 5 Optimal ilPPC model M1 can be linearly approximated by M2 and LIP but not by M3 and MSTd.

(a) Model M1 exhibited near-optimal behavior as the monkey. The psychophysical threshold under the combined condition (green) was indistinguishable from the Bayesian optimal prediction (black). (b) Ramping activity of M1 computed as the difference of PSTHs for IN and OUT trials. Activities from hypothetical units in the LIP layer with preferred direction close to $\pm 90^{\circ}$ were averaged together (see Figure 4c and Methods). Since M1 is optimal and homogeneous, we refer to M1's activities as "optimal traces" (see the main text). Notations are the same as before. (c) Optimal traces from M1 (thick shaded bands) can be linearly reconstructed by population activities obtained from a heterogenous model M2 (dashed curves). Model M2 had the same network architecture as M1 except that it relies on random combinations of ilPPC inputs in the integration layer (see Methods). (d) Optimal traces can also be linearly reconstructed by heterogenous single neuron activities from the LIP data. The similarity between $\mathbf{c}$ and $\mathbf{d}$ suggests that both model M2 and monkey LIP are heterogeneous variations of to the optimal ilPPC model M1. (e and f) In contrast, the optimal traces cannot be reconstructed from activities of a suboptimal model M3 (e) or from the MSTd data (f), presumably because the time constants in M3 and MSTd were too short. (g) Mean squared error of the fits in panels $\mathbf{c}-\mathbf{f}$. Error bars and $p$ values were from subsampling test ( $n=50$ neurons, 1000 times). (h) Normalized readout weights ordered by magnitude. Shaded error bands indicate standard deviations of the subsampling distributions. (i) The kurtosis of the distributions of weights. The black curve in (h) and black bar in (e) were from random readout weights (see Methods). 

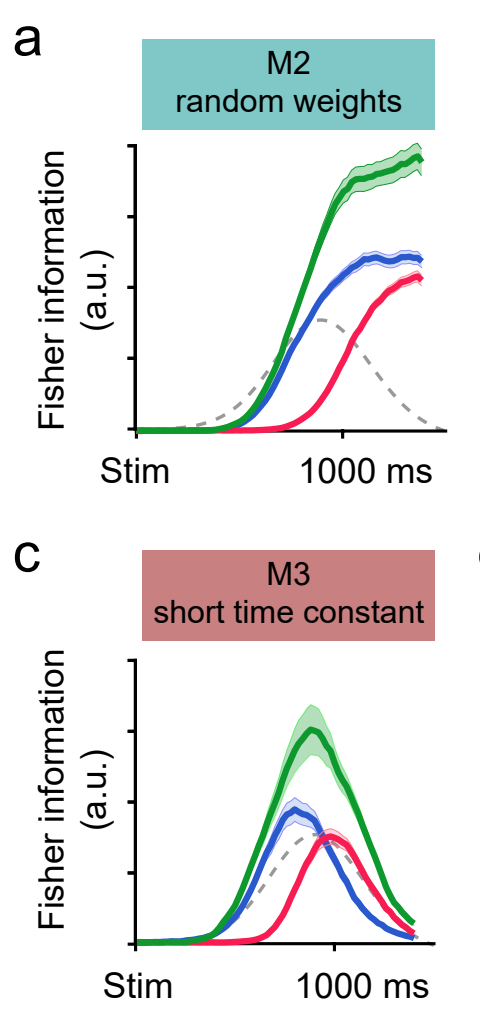

b

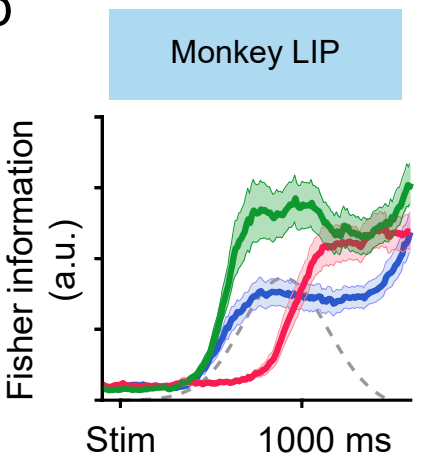

d

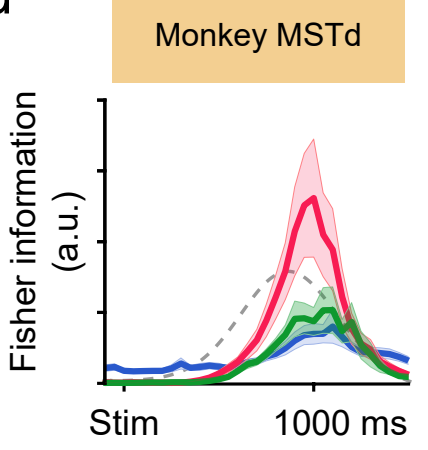

Figure 6 Shuffled Fisher information for the model and the experimental data.

(a) Shuffled Fisher information of M2 calculated by $I_{\text {shuffled }}=\sum_{i} f_{i}^{\prime 2} / \sigma_{\mathrm{i}}^{2}$, where $f_{i}{ }^{\prime}$ denotes the derivative of the local tuning curve of the $i$ th neuron and $\sigma_{i}^{2}$ denotes the averaged variance of its responses around $0^{\circ}$ (see Methods). Both correct and wrong trials were included. Shaded error bands, s.e.m. estimated from bootstrap. Note that the absolute value of shuffled Fisher information is arbitrary. (b-d) Same as in a but for the monkey LIP data, the M3 responses, and the monkey MSTd data, respectively. Note that LIP is similar to M2, and MSTd to M3. 